\title{
Detecting alkali-silica reaction: a multi-physics approach
}

Mehdi Rashidi ${ }^{\mathrm{a}}$, Marc C. Knapp ${ }^{\mathrm{a}}$, Ashkan Hashemi ${ }^{\mathrm{b}}$, Jin-Yeon Kimª ${ }^{\mathrm{a}}$ Kristen Donnell ${ }^{\mathrm{b}}$, Reza Zoughi $^{\text {b }}$, Laurence J. Jacobs ${ }^{\mathrm{a}, \mathrm{c}}$, Kimberly E. Kurtis ${ }^{\mathrm{a}, *}$

${ }^{a}$ School of Civil and Environmental Engineering, Georgia Institute of Technology, 790 Atlantic Drive, Atlanta, GA 30332, USA.

${ }^{\mathrm{b}}$ Department of Electrical and Computer Engineering, Missouri University of Science and Technology, Rolla, MO 65409, USA

${ }^{c}$ G. W. Woodruff School of Mechanical Engineering, Georgia Institute of Technology, 813 Ferst Drive, Atlanta, GA 30332

* Corresponding author: kimberly.kurtis@ce.gatech.edu, telephone: +1 4043850825

\section{Abstract}

A multi-physics approach for the assessment of alkali silica reaction (ASR) generates new foundational understanding of the nature of the reaction, which ultimately can be used for the development of techniques and tools for the assessment and monitoring of existing concrete structures. The approach combines two nondestructive evaluation techniques: (1) nonlinear acoustic measurements, which are sensitive to microcracking; and (2) microwave materials characterization measurements, which are sensitive to moisture including the transition of water from its free state in the pore solution to a bound state within accumulating ASR gel. Comparison with assessment of expansion and damage rating index obtained from petrographic analysis on standard mortar bars shows a correlation between all of the measures. Specifically, a strong correlation is found between the cumulative average nonlinearity parameter and expansion, and there is also agreement of the microwave measurements with the damage rating index.

Keywords: Alkali-Aggregate Reaction; Microwave; Nonlinear Acoustics; Characterization; Expansion; Damage 


\section{Introduction}

One of the most recognizable indications of the alkali-silica reaction (ASR) is gel exudation at the concrete surface $[1,2]$. Once the gel reaches the surface of a structure, however, extensive expansion and damage limit repair options and potentially deleterious materials may have been used in subsequent construction projects. Therefore, early detection of alkali-silica reaction occurring within concrete would be a significant contribution to the sustainability of concrete infrastructure.

The primary cause of deterioration associated with the ASR is the expansion of a characteristic gel that generates internal tensile stress and leads to microcracking. While it is understood that the gel forms from the reaction between aggregate containing amorphous or poorly crystalline siliceous minerals and the alkaline pore solution in concrete, the underlying mechanism of expansion and swelling pressure remains poorly understood [3-5]. Also, the relationship between the ASR gel composition, the volume of gel produced, and the potential for damage in concrete remain unresolved [3-7]. From a fundamental perspective, a comprehensive understanding of the relationships among gel composition, gel volume, gel expansion, and rate and extent of damage to concrete structures is critical for advancing the state of the art, and would have important implications for the screening of materials, the validation of mix designs, and the monitoring of concrete infrastructure.

Here, a multi-physics approach is used to provide a comprehensive study of ASR which aims to link chemical changes (i.e., transition of water from pore solution into ASR gel), physical changes (i.e., gel formation, microcracking) and mechanical properties (i.e., changes in material 
linear elasticity or increasing nonlinearity with increasing damage). This approach includes monitoring of progressive ASR damage and its relationship with water absorption using nonlinear acoustic and microwave nondestructive testing methods, with validation by standard expansion measurements and petrographic assessment.

In recent years, the nondestructive evaluation of ASR has developed significantly. For instance, Chen et al. [8] introduced an acoustic technique, the nonlinear impact resonance acoustic spectroscopy (NIRAS) method, to quantify material nonlinearity induced by ASR damage, demonstrating that nonlinear techniques are more sensitive to changes in microstructures than conventional linear methods. Over time, several studies have applied linear and nonlinear ultrasonic techniques to quantify ASR damage in concrete [9-11] and to assess the potential reactivity of certain aggregate [8]. Ultimately, this research has led to the adoption of a provisional standard in AASHTO [12] for the screening of aggregate and concrete mix designs in conjunction with standard concrete prism testing (ASTM C 1293 [13]).

While nonlinear acoustic methods are reliable for detecting microcracks [14-17] which may stem from a variety of sources in cement-based composites, such measurements cannot definitively detect ASR in concrete because they cannot directly detect the presence of ASR gel. In contrast, microwave measurements, which are sensitive to the amount of water and the chemical state of water in a material $[18,19]$ have been applied to investigate the water-tocement ratio and the progression of chloride ion ingress and quantifying defects in cement-based materials $[18,20]$. Recently, preliminary studies have demonstrated the potential for microwave measurements to distinguish between mortars containing alkali-silica reactive aggregate and those containing nonreactive aggregate [21, 22], and mortars kept indifferent environmental conditions [23]. 
The current research explores the combination of emerging microwave materials characterization measurements with proven nonlinear acoustic measurements and verifies both nondestructive techniques against expansion and petrographic results. Relating the results from four distinct measures of ASR provides new knowledge about the connection between the formation of the ASR gel and the evolution of ASR damage.

\section{Background}

\subsection{Microwave methods}

Materials can be characterized by their intrinsic complex relative (to free-space) dielectric constant, $\varepsilon_{r}$, as represented in Eq. (1) [24]:

$\varepsilon_{r}=\varepsilon_{r}^{\prime}+j \varepsilon_{r}^{\prime \prime}$,

where $\varepsilon_{r}^{\prime}$, the relative permittivity, represents the ability of a material to store energy, and $\varepsilon_{r}^{\prime \prime}$, the relative loss factor, represents the ability of a material to absorb energy. Since the values of relative permittivity and loss factor are normalized to those of free-space $\left(\varepsilon_{0}\right)$, they are dimensionless. By passing electromagnetic waves through a sample and measuring the reflected and transmitted energy, the dielectric properties of a material can be evaluated. In general, the dielectric properties of a consolidated material (i.e., concrete) are controlled by the dielectric properties of individual components, their relative proportions, and the physical and chemical properties of the material [25]. Dielectric properties are also frequency dependent, and certain frequencies of microwaves in particular are more sensitive to changes in the chemical composition of mortar and concrete. Based on previous work, microwave signals at the S-band frequency range (2.6-3.95 GHz) have shown high sensitivity to changes in mortars undergoing the alkali-silica reaction [21] and have been capable of distinguishing between the potentially 
reactive and nonreactive aggregate in the mortar samples by providing temporal data on the microstructural evolution [22]. In this research, S-band frequency range is used to assess mortars containing aggregate of varying reactivity at the end of accelerated mortar bar testing (AMBT) [26]. Differences in the reactivity of aggregate at the end of the test cause variations in the amounts of ASR gel, water content, and water state — bound or free water — in the mortars, which can be detected by differences in their dielectric properties. By combining data on dielectric properties with test methods that can detect the evolution of microcracking, a new test procedure that specifically detects ASR damage may be developed.

\subsection{Nonlinear acoustic methods}

Distributed microcracking caused by ASR can be detected by nonlinear acoustic methods [89 11, 27]. While linear acoustic methods (e.g., ultrasonic pulse velocity method) detect macroscale deficiencies $[8-11,28,29]$, nonlinear acoustic methods are more sensitive to the presence of microcracks and therefore are better tools for detecting incipient ASR damage [8-9, 11]. Nonlinear acoustic techniques have been used for the damage characterization of a variety of materials, whose nonlinear stress-strain relationship can be represented in Eq. (2) $[30,31]$ as

$$
\sigma=E_{0}\left[\varepsilon+\beta \varepsilon^{2}+\delta \varepsilon^{3}+\alpha\left\{\varepsilon(\Delta \varepsilon)+\frac{1}{2} \operatorname{sgn}(\dot{\varepsilon})\left(\varepsilon^{2}-(\Delta \varepsilon)^{2}\right)\right\}\right]
$$

Where $\sigma$ is longitudinal stress, $\varepsilon$ strain, $E_{0}$ the linear elastic modulus, $\beta$ the quadratic nonlinearity parameter, $\delta$ the cubic nonlinearity parameter, $\alpha$ the parameter representing the material hysteresis nonlinearity, $\Delta \varepsilon$ the strain amplitude, $\dot{\varepsilon}$ the strain rate, and $\operatorname{sgn}(\dot{\varepsilon})$ the signum function where $\operatorname{sgn}(\dot{\varepsilon})=1$ if $\dot{\varepsilon}>0, \operatorname{sgn}(\dot{\varepsilon})=-1$ if $\dot{\varepsilon}<0$, and $\operatorname{sgn}(\dot{\varepsilon})=0$ if $\dot{\varepsilon}=0$. Since microcracking is the main cause of nonlinearity in cement-based materials, the hysteresis 
nonlinearity parameter $(\alpha)$ dominates the elastic nonlinearity parameters $(\beta, \delta)[31]$. Therefore, the extent of damage or microcracking can be represented by the magnitude of hysteresis nonlinearity.

In standard mortar bars (such as those examined here) or in concrete prisms with hysteresis nonlinearity, the resonance frequency of a sample is the function of the excitation amplitude. As the excitation amplitude increases, the resonance frequency shifts to a lower value [11] and as damage progresses, the increase in the excitation amplitude causes a greater downward shift in the resonance frequency, which may be assessed using the nonlinear impact resonant acoustic spectroscopy (NIRAS) method, [8, 11, 31-35] and the hysteresis nonlinearity can be evaluated according to Eq. (3), as

$\frac{f_{0}-f}{f_{0}}=\alpha^{\prime} A$

where $f_{0}$ is the linear resonant frequency of the sample, $f$ the resonant frequency of the sample at the excitation level, $\alpha^{\prime}$ directly proportional to the hysteresis nonlinearity parameter $(\alpha)$ and $A$ the acceleration amplitude.

\section{Experimental Investigation}

Standard mortar bars were prepared using three aggregate sources: two potentially reactive aggregate (Reactive-1 and Reactive-2) and one nonreactive aggregate (Non-Reactive). Samples were subjected to curing and exposure prescribed by the accelerated mortar bar test (AMBT) as in ASTM C 1260 [26]. Subsequently, expansion, petrographic, microwave, and nonlinear acoustic assessments were performed on the samples. The sample exposure was consistent across all tests so that test results could be directly compared. 


\subsection{Materials and sample preparation}

Based on the potential reactivity level as defined in ASTM C 1260 [26] and field performance history, three sources of natural aggregate (Table 1) from the United States were selected. Aggregate materials were graded such that 10 percent of the mass was retained on a number $8(2.36 \mathrm{~mm})$ sieve, 25 percent on a number $16(1.18 \mathrm{~mm})$ sieve, 25 percent on a number $30(600 \mu \mathrm{m})$ sieve, 25 percent on a number $50(300 \mu \mathrm{m})$ sieve, and 15 percent on a number 100 $(150 \mu \mathrm{m})$ sieve. Aggregate used in this study were not washed.

Mortars were cast from each of these sands with the same ASTM C150 Type I cement (Lehigh Cement Company, LLC). The cement Bogue composition was $42.72 \% \mathrm{C}_{3} \mathrm{~S}, 24.77 \% \mathrm{C}_{2} \mathrm{~S}, 6.29 \%$ $\mathrm{C}_{3} \mathrm{~A}$, and $12.23 \% \mathrm{C}_{4} \mathrm{AF}$ and the equivalent soda content $\left(\mathrm{Na}_{2} \mathrm{O}_{\mathrm{e}}\right)$ was $0.80 \%$.

Table 1. Aggregate identification

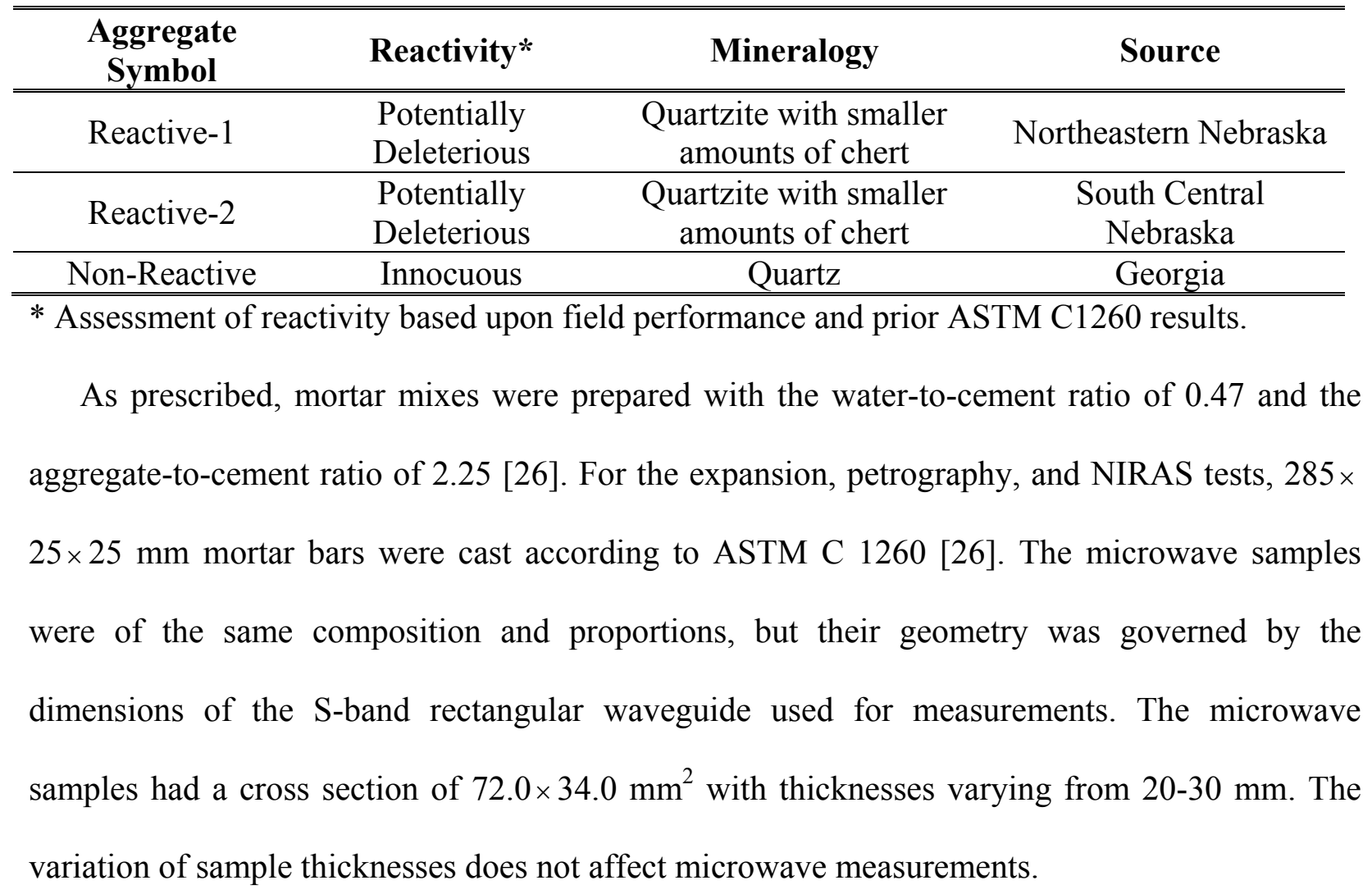




\subsection{Exposure and expansion measurements}

Mortar bars were demolded 24 hours after casting, kept in deionized water at $80^{\circ} \mathrm{C}$ for the next 24 hours, and subsequently submerged in $1 \mathrm{~N} \mathrm{NaOH}$ solution at $80^{\circ} \mathrm{C}$ for fourteen days, with expansion and nonlinearity measured periodically [26]. For the microwave measurements, two sets of samples were prepared. One of the sample sets was exposed to the same exposure type as the mortar bars, while the other sample set was submerged in deionized water at the temperature of $80^{\circ} \mathrm{C}$ after demolding. Comparison between the water-exposed and sodium hydroxide-exposed sets allowed for discrimination between the effects of cement hydration and high temperature curing at the alkaline solution and those of the ASR.

\subsection{Petrographic assessment using the Damage Rating Index}

The evaluation of damage using petrographic analysis in the ASR-affected concrete samples is generally performed by the calculation of damage rating index (DRI), which assigns weights to the different types of defects, as shown in Table 2.

Table 2. Defect scaling factors used for DRI [36, 37]

\begin{tabular}{cc}
\hline Defect Type & Scaling Factor \\
\hline $\begin{array}{c}\text { Crack in coarse aggregate } \\
\text { filled with gel }\end{array}$ & 2 \\
\hline $\begin{array}{c}\text { Crack in coarse aggregate } \\
\text { without gel }\end{array}$ & 0.25 \\
\hline $\begin{array}{c}\text { Crack in cement paste filled } \\
\text { with gel }\end{array}$ & 4 \\
\hline $\begin{array}{c}\text { Crack in cement paste without } \\
\text { gel }\end{array}$ & 2 \\
\hline Reaction rim & 0.5 \\
\hline Air void with gel & 0.5 \\
\hline \hline
\end{tabular}

To perform the DRI, a section of concrete with the thickness of about $20-30 \mathrm{~mm}$ is cut from a cylinder or prism concrete sample. The section is polished, and grid sizes of $15 \times 15 \mathrm{~mm}^{2}$ 
are drawn on the sample surface. In each grid, the number of each defect type is counted at the magnification of 16x, multiplied by its weighting factor, and added to each other. The final value is reported per $100 \mathrm{~cm}^{2}[38]$.

While DRI is generally performed on concrete experiencing ASR in the field [36] or under ASTM C1293 [13] exposure, an extension of this quantitative image assessment approach was attempted to evaluate the extent of damage in mortars. To this end, those defect types that related to the coarse aggregate — crack in the coarse aggregate with and without gel—were assigned to the fine aggregate — crack in the fine aggregate with and without gel—while their scaling factors maintained the same. Furthermore, the grid size and magnifications were modified to maintain proportionality to the aggregate size and ASR-induced defects. In concrete, since coarse aggregate contributes to a significant portion of the microstructure, a large grid size of $15 \times 15 \mathrm{~mm}^{2}$ is needed. However, in mortar, because the microstructure is quite fine, the grid size is decreased to $2 \times 2 \mathrm{~mm}^{2}$. Furthermore, the magnification is increased from $16 \mathrm{x}$ in concrete to $25 \mathrm{x}$ in mortar for better observation of microstructural defects.

DRI values at days six, ten, and fourteen of exposure were calculated. At each age, the same sample of each sample set was used for the petrographic examination. To preserve and quantify regions containing gel, highlight cracks, and allow discrimination between defects containing gel and those without gel, sample preparation and imaging were performed in two stages $[36,37]$. In the first stage, samples were cut from the mortar bars with a low speed, ethanol-cooled saw. Then, they were polished to 320 grit with ethanol as a lubricant. Polishing samples was limited to minimize the removal of gel, while still allowing high quality images to be obtained. Afterwards, samples were stained with uranyl acetate to increase the visibility of the ASR gel [39] following the procedure described in [40]. The gel was observed using optical 
microscopy (Leica DMRE) under a mercury lamp light source. At each age, four sections were cut from each sample type and four images per section were obtained. Overall, sixteen images per sample type per age were taken. Subsequently, grids were overlain on the images and defect counting on two grids per field of view was performed to determine the number of gel-associated defects. Gel-associated defect categories included cracks in aggregate filled with gel, cracks in cement paste filled with gel, the reaction rim, and air void filled with gel. In the second stage, after washing to remove stain, samples were vacuum-impregnated with epoxy and polished to 600 grit with water as a lubricant. Images were again acquired under a mercury lamp light source, identical grids to those used in the first stage were overlain on images, and the defects were counted on two grids per field of view. Counting of defect types in the stage one and two was performed on the identical field of view. Defects without gel—cracks in aggregate without gel and cracks in cement paste without gel - were those observed in stage two but not in the stage one. Then, the total number of each defect type per grid area was calculated for each sample type. Subsequently, an appropriate scaling factor (as shown in Table 2) was applied to each defect type. DRI values were reported as the average number of defects per $100 \mathrm{~cm}^{2}$ of grid area [36].

\subsection{Microwave measurement experimental procedure}

Microwave measurements were made by inserting a mortar sample into the waveguide, connecting both ends of the waveguide to calibrated ports of an Agilent $8510 \mathrm{C}$ vector network analyzer (VNA) using coaxial cables, and recording the transmission coefficient $\left(S_{21}\right)$ and reflection coefficient $\left(S_{11}\right)$ of a signal (Fig. 1), from which the relative permittivity and loss factor can be calculated following the procedure outlined by Bois, et al. [41]. Since $\mathrm{S}_{11}$ and $\mathrm{S}_{21}$ 
interact with the sample, the calculated values are sensitive to physical and chemical properties of the sample such as the presence and state of water in mortar [22].

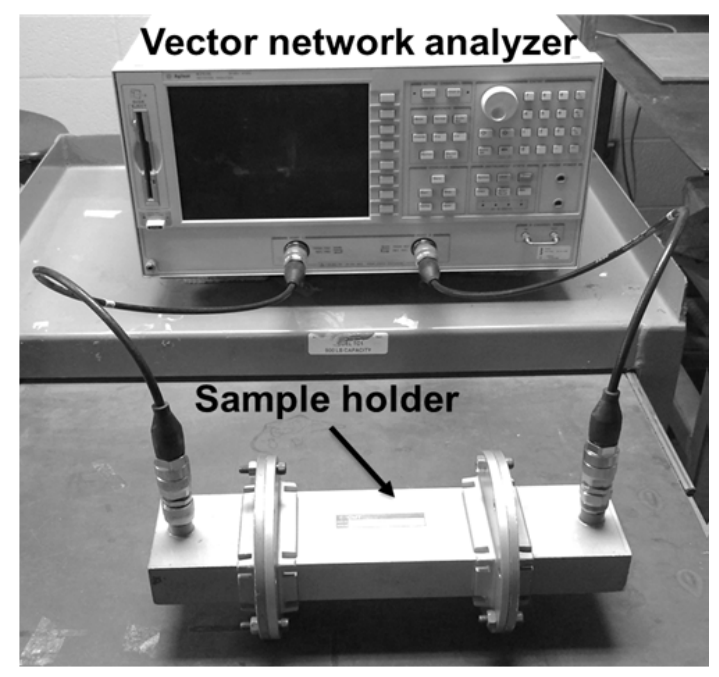

Fig. 1. Test setup for the microwave measurements.

\subsection{NIRAS experimental procedure}

NIRAS measurements were made by applying small impacts but with a minutely increasing force to the midpoint of the sample, capturing the response of the sample with an accelerometer located at the end of the sample, and recording the transient vibration response of the sample in the time domain with an oscilloscope, as shown in Fig. 2. Details on the experimental setup have been reported by Lesnicki et al. [11,32]. Later, the sample response to those impacts was analyzed in the frequency domain and $\alpha^{\prime}$ was calculated according to Eq. (3). 


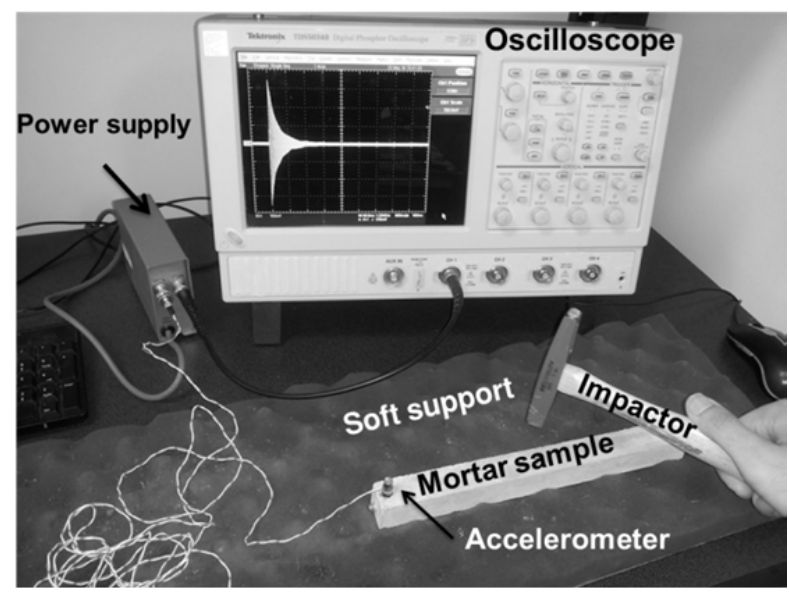

Fig. 2. NIRAS test setup.

Prior to the first nonlinear acoustic measurement on the mortar bars, NIRAS measurements on a linear elastic specimen (i.e., an aluminum bar) matching the geometry of the mortar samples were used to confirm the linearity of the experimental setup.

\section{Results}

This study aims to investigate the alkali-silica reaction using a multi-physics approach, using methods based upon mechanics and electromagnetism. Specifically, the effect of accumulating ASR on the mechanical properties of mortar are monitored with NIRAS measurements and the change in the electromagnetic properties are monitored with the microwave measurements, which measure the dielectric constants of the samples. Furthermore, the expansion of mortar bars prepared according to the AMBT and quantitative assessment of changes in material structure are used as baseline data.

In the following results, the length of an error bar is equal to one standard deviation. Moreover, the Pearson product-moment correlation coefficient [42] - equivalent to the $\mathrm{R}$ of best linear fit - is used to examine the correlation between the mean values of two data sets.

\subsection{Expansion}


The expansion of mortar bars, measured daily during the fourteen day of AMBT exposure, are shown in Fig. 3. By ASTM C 1260 [26], both Reactive-1 and Reactive-2 aggregate are deemed potentially deleterious, and the Non-Reactive aggregate as innocuous. Reactive-1 samples show the largest expansion, nearly $0.4 \%$, at the end of the test period. Moreover, the expansion rate of those samples are almost constant from day three to day fourteen, which suggests the high availability of reactive silica. In contrast to the Reactive-1 samples, the expansion of the Reactive-2 samples starts at the day one of AMBT and exceeds the expansion of the Reactive-1 samples between day two and day nine. However, the rate of expansion observed for the Reactive-2 samples decreases from day seven, which suggests a decrease in the availability of reactive silica. In contrast to reactive samples, the expansion of Non-Reactive samples starts at day two of AMBT. By day five, the Non-Reactive samples experience half of their fourteen-day expansion. Afterwards, samples expand at a low rate and their expansion reaches a plateau at day twelve.

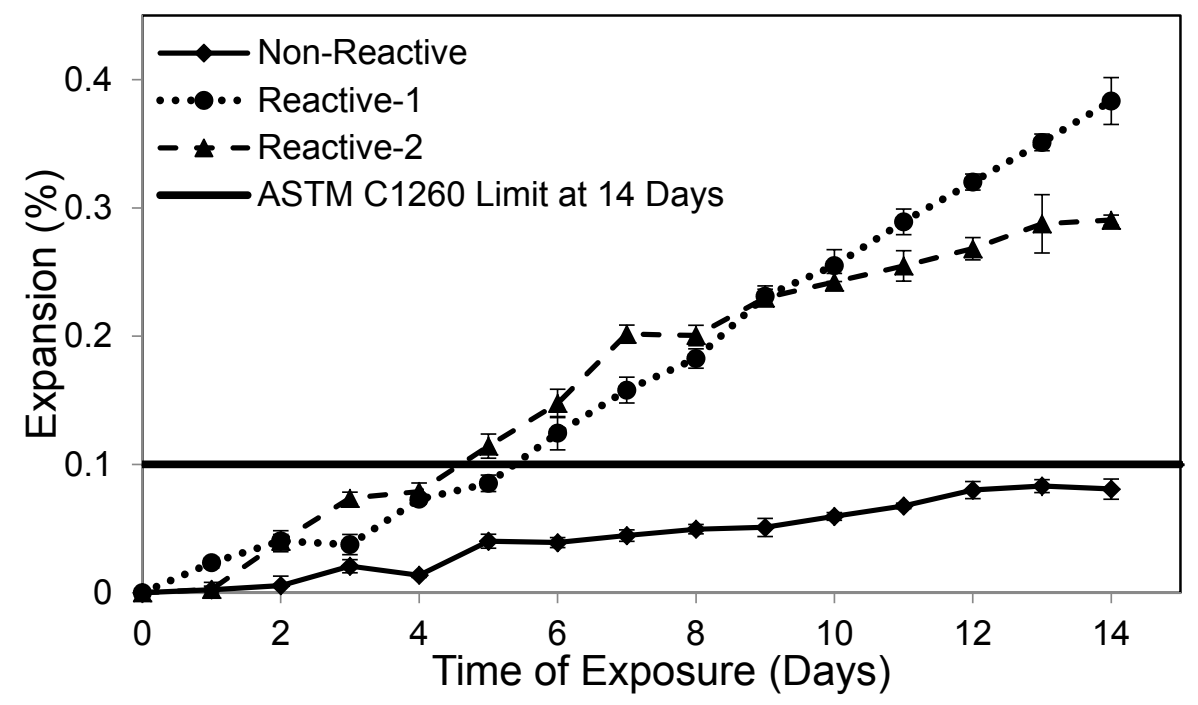

Fig. 3. AMBT expansion results.

\subsection{Petrography}


Representative fluorescence microscopy images of each of the three mortar types after fourteen days of exposure are shown in Fig. 4, while Fig. 5 shows representative images of epoxy impregnated mortar samples at the same age. The fluorescence images show cracking around and through the aggregates and also in the paste for the potentially reactive samples; there is also a greater concentration of fluorescence, likely due to the presence of gel, around the aggregates and in some of the cracks. In addition, the fluorescence of some of aggregates even without cracking is potentially caused by the high $\mathrm{pH}$ of the $\mathrm{NaOH}$ soaking solution, which facilitates the ion exchange of sodium and uranyl at the silica surface [39].

a.)

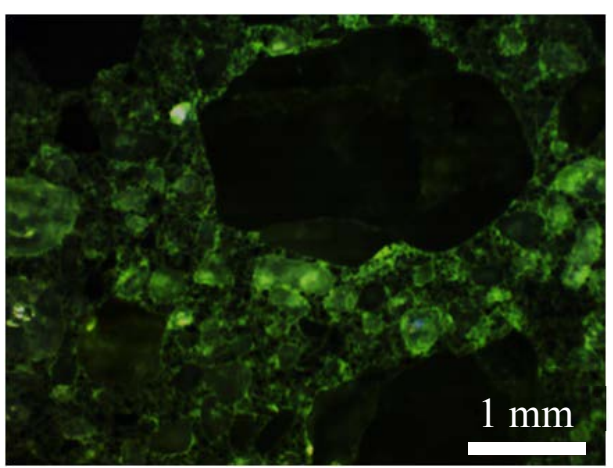

c.)

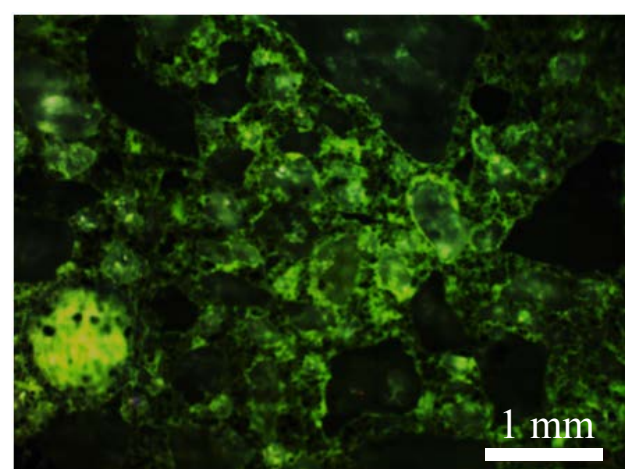

b.)

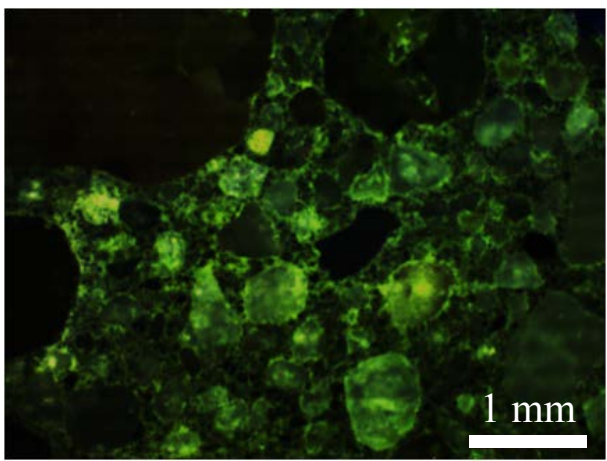

Fig. 4. Fluorescence of gel a.) Reactive-1 sample, b.) Reactive-2 sample, c.) Non-Reactive sample. The samples were exposed to AMBT conditions for fourteen days. 
a.)

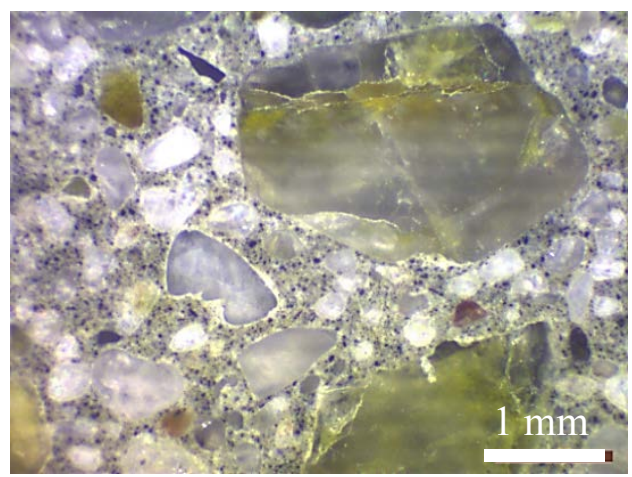

c.)

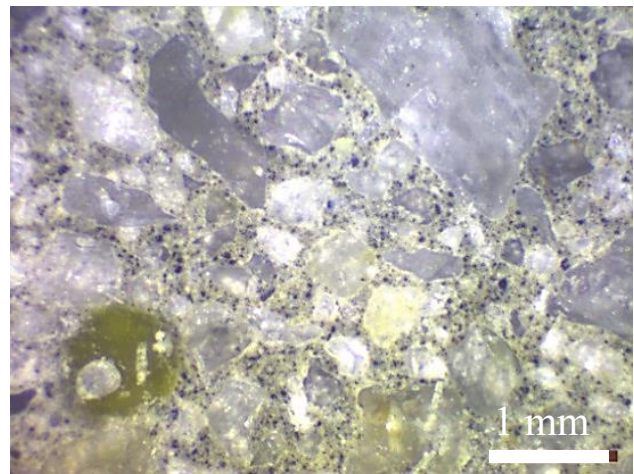

b.)

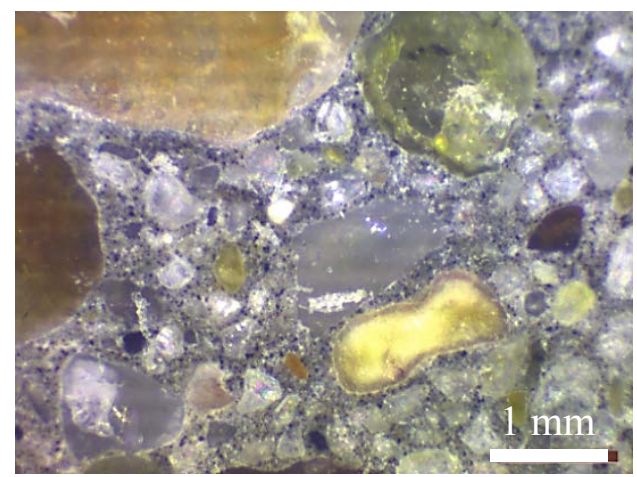

Fig. 5. Epoxy impregnated sample a.) Reactive-1 sample, b.) Reactive-2 sample, c.) NonReactive sample. Samples were exposed to AMBT conditions for fourteen days.

DRI values, obtained at six, ten, and fourteen days of exposure during AMBT, are shown in Fig. 6. Overall, the DRI values reported here for mortars are significantly larger than those previously reported in concrete; this likely stems from the aggressive nature of the AMBT compared to field exposure and also the smaller aggregate size in mortars compared to concrete. In other words, since in the calculation of DRI for mortar samples, defects associated with coarse aggregates in concrete were assigned to those with fine aggregate in the mortar, a larger number of defects per $100 \mathrm{~cm}^{2}$ are observed in the mortar samples compared to concrete samples having the same geometry and exposure condition. However, if the number of defects per $100 \mathrm{~cm}^{2}$ is scaled down to be proportional to the ratio of the size of the grids used in the mortar and concrete $\left(\frac{2 \times 2 \mathrm{~mm}^{2}}{15 \times 15 \mathrm{~mm}^{2}}=0.0178\right)$, then the DRI values for the $1.78 \mathrm{~cm}^{2}$ of mortar samples are in the 
range of 57 to 137 , which is comparable to the DRI values reported for the $100 \mathrm{~cm}^{2}$ of concrete $[37,38]$.

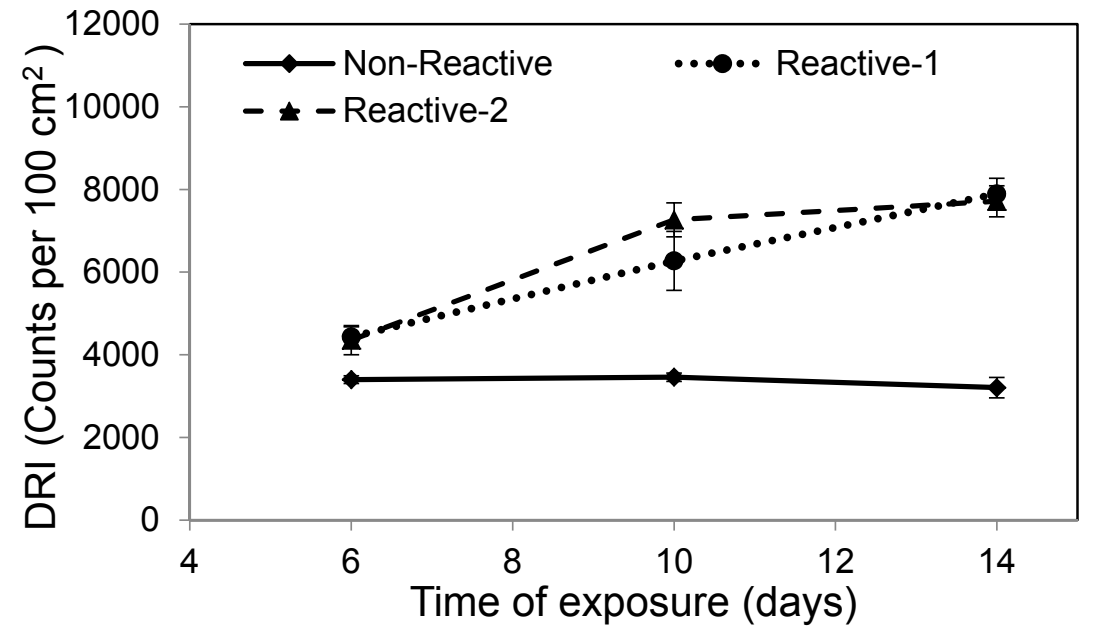

Fig. 6. DRI values at different ages.

The DRI values do distinguish between potentially deleterious (i.e., Reactive-1 and Reactive-2) aggregates whose mortars show more damage and innocuous (i.e., Non-Reactive) samples. However, it is difficult to differentiate the level of damage between Reactive-1 and Reactive-2 samples; DRI values of Reactive-1 and Reactive-2 samples are almost identical at day six and day fourteen. At day ten the average DRI value of Reactive-2 samples is larger than that of Reactive-1 samples, which has a large standard deviation.

To investigate the significance of the difference between the DRI values of the two potentially reactive aggregate at day ten, hypothesis testing is performed. The test statistically differentiates between those for the p-value of equal or bigger than 0.15 (a typical p-value is 0.01 or 0.05$)$. (Details on the hypothesis testing are shown in the next sections.) Although the DRI values for the Recative- 1 and Reactive- 2 samples may not be statistically different, their average DRI values follow different trends. The average DRI value and expansion data increases linearly for the Reactive-1 samples, but for the Reactive-2 samples, the increase in the average DRI value 
from day ten to fourteen is less than the increase from day six to ten. This is similar to the trend also observed in the expansion data of the Reactive- 2 samples. The DRI values of the NonReactive samples do not increase with the time of exposure, as would be expected.

Furthermore, Fig. 7 shows the correlation between DRI values and the expansion data at days six, ten, and fourteen of the AMBT. DRI and the expansion data for potentially reactive samples increase more consistently than for the Non-Reactive samples. In addition, the slope of the best linear fit to data of each sample-type represents the increment of damage per unit expansion and it is different for the three sample types. The slope is larger for the Reactive-2 than the Reactive-1 samples suggesting more damage in the Reactive-2 samples than the Reactive-1, at the same expansion level. Moreover, the slope of the best fit for the Non-Reactive aggregate is slightly negative.

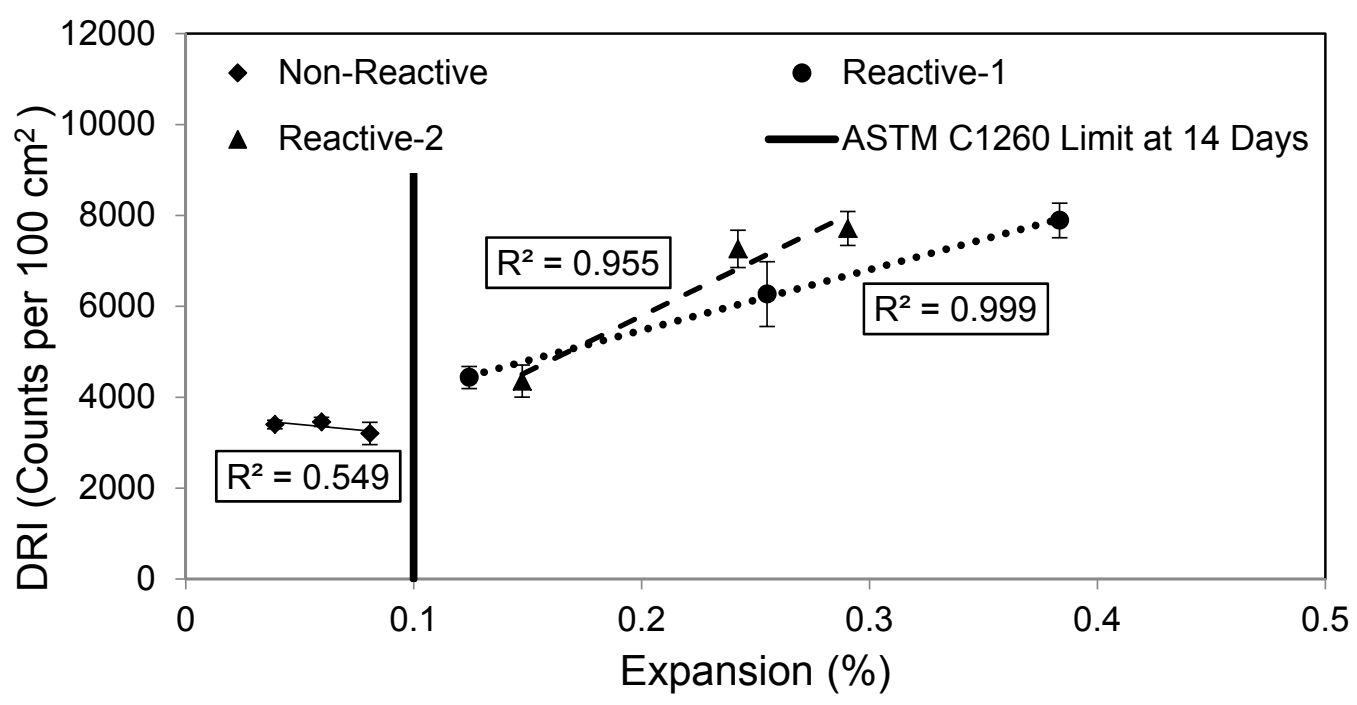

Fig. 7. Correlation between DRI values and expansion data for the three types of aggregate. The expansion data and DRI values were measured at the day six, ten and fourteen of AMBT.

The DRI values may be used to screen ASR reactive aggregates. Considering the relationship between the DRI values and the fourteen-day expansion of sample types, potentially reactive 
samples experience greater DRI values and expansion than the Non-Reactive samples. While an increase in DRI is associated with increased fourteen-day expansion, the relationship between those two values is not linear. However, a limit could be established to screen the potentially reactive aggregates and nonreactive aggregates based on the damage estimated by the DRI.

\subsection{Microwave measurements}

Dielectric properties of mortar samples exposed to the sodium hydroxide solution were measured and compared to measurements of those of companion samples exposed to water. This approach allows for changes due to ASR to be separated from those due to cement hydration, while maintaining consistency with ASTM C1260 methods used for expansion, DRI, and NIRAS measurements.

Considering the average value of measurements, the relative permittivity of the samples exposed to the sodium hydroxide solution is greater than those exposed to water for all three cases, as shown in Fig. 8. However, the difference between the relative permittivity of the NonReactive samples exposed to water and sodium hydroxide solution is very minor. That difference

includes the effect of cement hydration during curing at high temperature and exposure to alkali solution, which affects the pore solution composition and also potentially leads to subtle changes in the microstructure. So, it can be concluded that the cement hydration during curing and exposure to the alkaline solution has a relatively small effect on relative permittivity values in the absence of ASR. Furthermore, both potentially reactive aggregate mortars, when soaked in water, have similar dielectric properties to the Non-Reactive case demonstrating that the alkali concentration is not sufficient for ASR. In contrast, the relative permittivity of Reactive-1 and Reactive-2 samples that are exposed to the sodium hydroxide solutions are considerably greater than those exposed to water, since $\mathrm{NaOH}$ solution provides sufficient amount of alkalis for ASR. 
These results demonstrate that the presence of ASR gel can be detected by measurement of relative permittivity.

a.

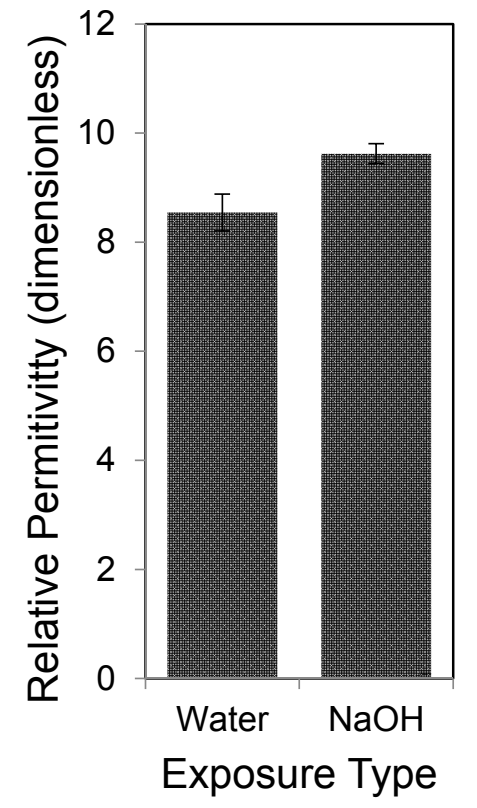

b.

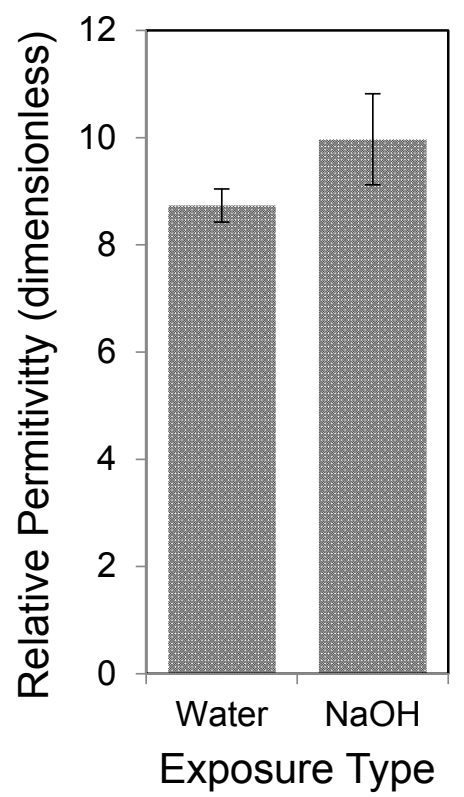

c.

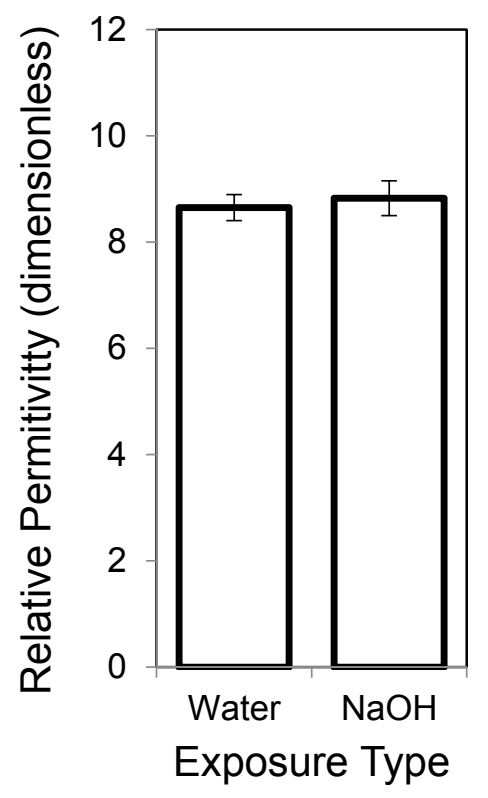

Fig. 8. The relative permittivity of samples with different fourteen-day exposure condition a.) Reactive-1 samples, b.) Reactive-2 samples, c.) Non-Reactive.

Two phenomena may contribute to the larger difference in relative permittivity of potentially reactive samples than that of Non-Reactive samples exposed to sodium hydroxide solution and water. First, for samples soaked in sodium hydroxide solution, the greater extent of cracking and damage in the Reactive-1 and Reactive-2 samples than Non-reactive samples, as evidenced by their greater expansion and their DRI values, increases the porosity of the samples. As a result, more sodium hydroxide solution, which has a high relative permittivity [43], enters the samples, and the overall relative permittivity of samples increases. In contrast, for samples soaked in water, since the alkali concentration is not enough for ASR and subsequent microcracking, the overall porosity is lower than those exposed to sodium hydroxide solution. As a result, less water enters the samples and their relative permittivity is lower than those exposed 
to $\mathrm{NaOH}$ solution. A second possible contributor to the increase in the relative permittivity of samples exposed to the alkali solution is the presence of ASR gel. Kirkpatrick, et al. [44] using molecular dynamics simulations and x-ray diffraction of ASR gels as confirmation, have shown that water molecules are accommodated between nano-particles within in the gel, indicating a high degree of binding of water within ASR gel. Therefore, the moisture bound within ASR gel is also expected to contribute to a greater relative permittivity.

Fig. 9 shows results for loss factor, comparing results for samples with each of the three aggregates. For all cases, those exposed to sodium hydroxide solution have a larger (more negative) loss factor than those kept in water. Examining the various influences on loss factor (e.g., the state of water, presence of cracks, and ionic concentration of pore solution) and comparing these with assessment of ASR-induced damage suggests that the change in ionic concentration of the pore solution caused by soaking in $\mathrm{NaOH}$ solution is the dominant cause of the trend observed. Ionic concentration has a significant effect on the loss factor, while it only slightly affects the relative permittivity [43]. This effect on the loss factor is more remarkable at low frequencies (i.e., $\mathrm{S}$ band frequency) and the evidence of that is the significant difference in the loss factor comparing samples exposed to water (where leaching occurs) with those exposed to the AMBT conditions. The ingress of sodium and hydroxyl ions from the surrounding solution is believed to dominate any potential changes in loss factor associated with ASR gel formation or resulting damage. 
a.

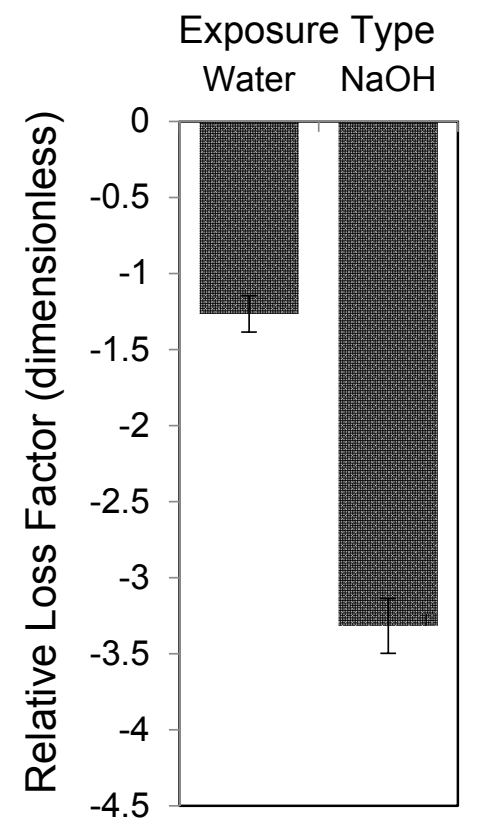

b.

Exposure Type

Water $\mathrm{NaOH}$

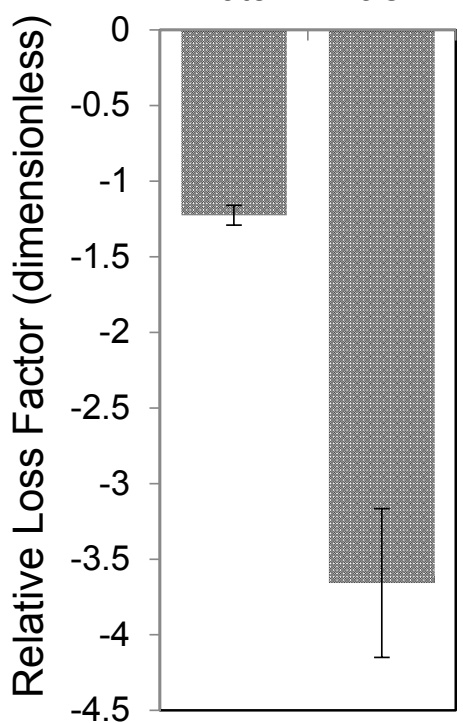

c.

Exposure Type

Water $\mathrm{NaOH}$

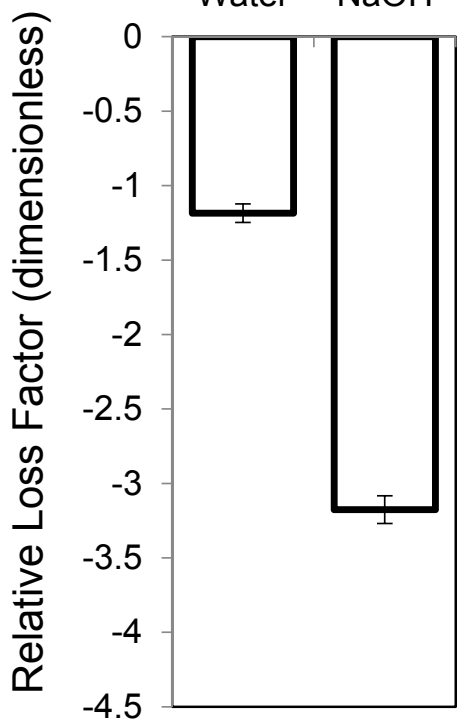

Fig. 9. The relative loss factor of samples with different fourteen-day exposure conditions. a.) Reactive-1 samples, b.) Reactive-2 samples, c.) Non-Reactive.

When compared to the influence of loss factor reported in previous investigation by Donnell, et al. [16,17] and Hashemi, et al. [45] in which the temporal measured loss factor showed stronger sensitivity to ASR gel formation than temporal relative permittivity, these results show relative permittivity is more sensitive to the presence of ASR gel. However, in those prior studies, mortar samples were not soaked in $\mathrm{NaOH}$ and water solution prior to performing the dielectric property measurements. Instead, during measurement period they were stored over water in a condition similar to ASTM C1293 test conditions [13]. When stored over water, relatively less free water enters the samples and the formation of ASR gel is the main cause of changes observed in the complex dielectric properties of mortars cast with potentially reactive aggregates. The differences in the findings of this study and previous ones are related to the exposure condition and temporal variations in sample properties, but remain consistent in terms of understanding parameters affecting dielectric properties of cement-based materials. 


\subsection{NIRAS measurements}

Results from the NIRAS measurements, performed on sodium hydroxide-exposed mortar bars daily, are shown in Fig. 10. The average nonlinearity parameter for each sample type represents the slope of the linear relationship between the downward resonance frequency shift and the excitation amplitude measured at that time for three replicates. That is, data for three replicate bars are merged and the nonlinearity parameter is calculated, which is a more conservative approach in the calculation of the average nonlinearity parameter than assuming three statistically independent random variables.

As observed from Fig. 10, the average nonlinearity parameter of the Non-Reactive mortar samples remains well below that of the potential reactive samples for the duration of testing. Early in the exposure (i.e., at $\sim 3$ days exposure and earlier), the Reactive- 2 samples exhibit the highest nonlinear behavior among aggregate types. Over time, however, the nonlinearity parameter of the Reactive-1 samples surpasses that of the Reactive- 2 mortars by day four. Moreover, the Reactive-1 samples exhibit the highest measured nonlinear behavior among aggregate types; this occurs at day six.

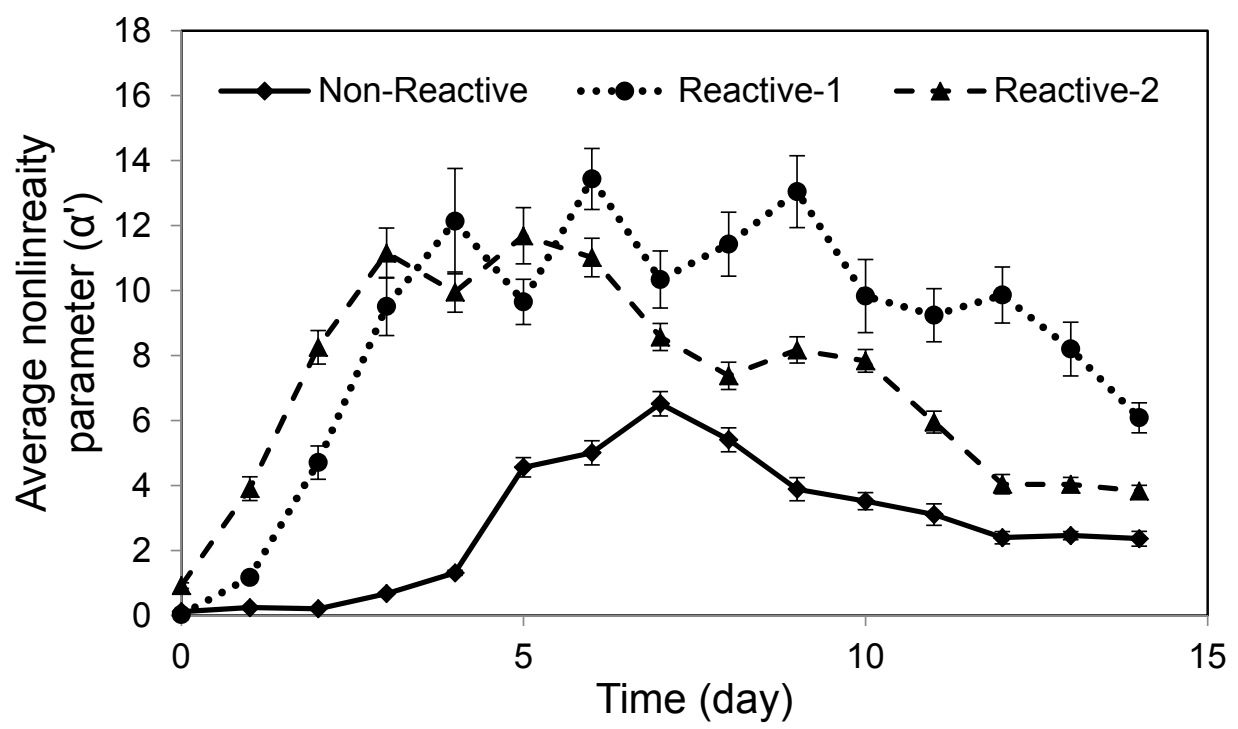


Fig. 10. Temporal average nonlinearity parameter.

The average nonlinearity parameters of all three mortars types show an oscillatory or up/down behavior; this trend in behavior is consistent with prior NIRAS measurements on ASRaffected mortar and concrete samples [8,11,32]. Initially, the nonlinearity increases as ASRinduced microcracking progresses. But, over time, the nonlinearity parameter decreases as mechanisms that reduce the nonlinearity—such as increased gel pressure in the microcracks, which restrains their surface asperity interaction [46] — dominate the potential increases in nonlinearity due to the generation of new microcracks. Interestingly, even the Non-Reactive samples follow the same pattern. This can be associated with the aggressive environment of AMBT, which induced expansion in the Non-Reactive aggregate but did not exceed the $0.1 \%$ threshold (Fig. 3). So, it is proposed that this inflection in nonlinearity parameter can be used as an indication of the potential for ASR damage. Specifically, it is hypothesized that samples showing higher variation in the nonlinearity parameter over the test period contain aggregate of greater potential reactivity. To test this hypothesis, the standard deviation of the average nonlinearity parameter for each source of aggregate is estimated as

$S_{\alpha^{\prime}}=\sqrt{\frac{\sum_{i=0}^{14}\left(\alpha^{\prime}(i)-\bar{\alpha}^{\prime}\right)^{2}}{14}}$

where $S_{\alpha^{\prime}}$ is the standard deviation of average nonlinearity parameter, $\alpha^{\prime}(i)$ average nonlinearity parameter at time $i$, and $\bar{\alpha}^{\prime}$ the average of $\alpha^{\prime}(i)$. The standard deviation of the average nonlinearity parameter is calculated and plotted against fourteen-day expansion as shown in Fig. 11a. A linear trend between those metrics, along with disparity between the cases for innocuous and potentially reactive aggregate sources, suggests the validity of this hypothesis. 
To further test this hypothesis, nonlinearity parameter and expansion data of four aggregate types are obtained from the work of Chen et al. [8]. Again, with these data, a linear trend between the standard deviation of the average nonlinearity parameter and expansion is found, as shown in Fig. 11b. It should be noted that, since the experimental setup and the calculated nonlinearity parameters in the Chen et al. [8] work differs from those in this studyChen et al. [8] normalized the average nonlinearity parameter to the initial average nonlinearity parameter while in this study the data is not normalized — the standard deviation of the average nonlinearity parameter between two studies cannot be directly compared. However, the slope of the linear correlation between the standard deviation of the average nonlinearity parameter and fourteen-day expansion in Fig. 11b is around 90\% of that slope in Fig. 11a. These results suggest that the increment of standard deviation of the average nonlinearity parameter per fourteen-day expansion of samples kept in the AMBT condition is approximately the same, despite the differences in the experimental setup and reported nonlinearity parameters.

a.)

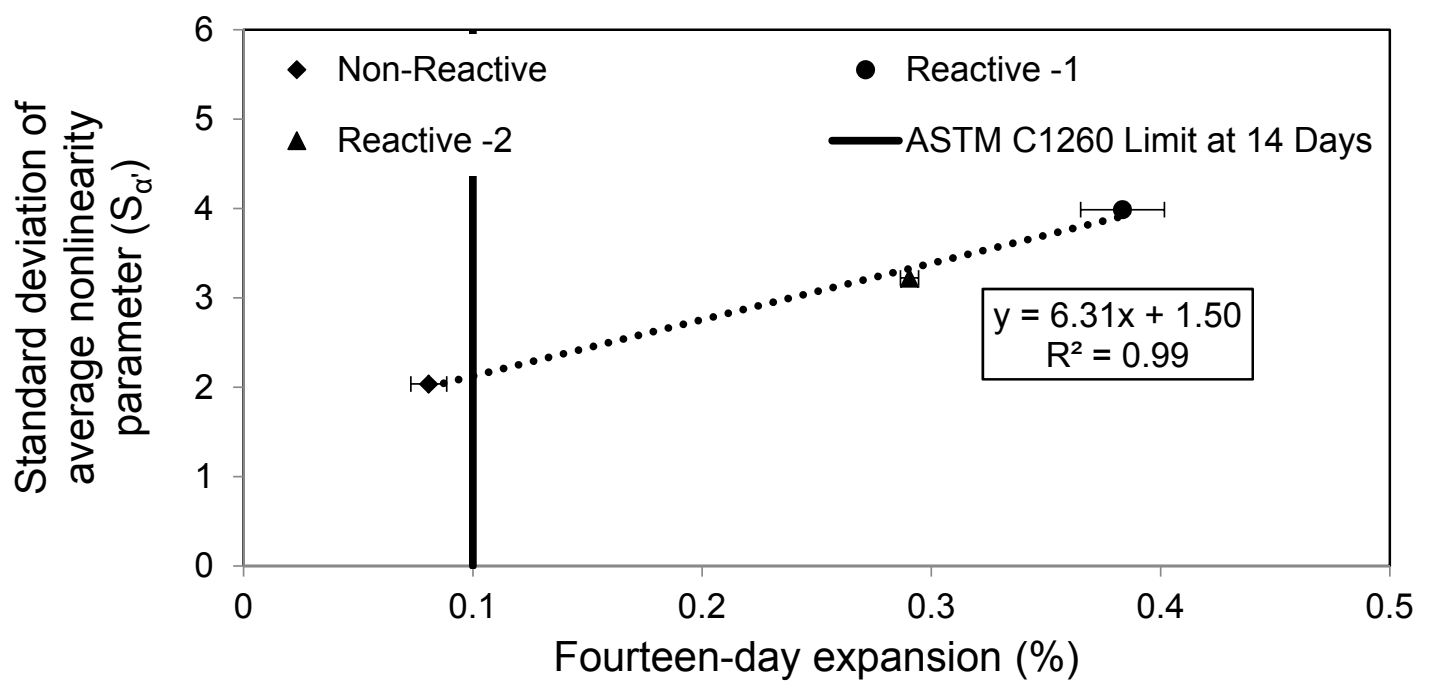


b.)

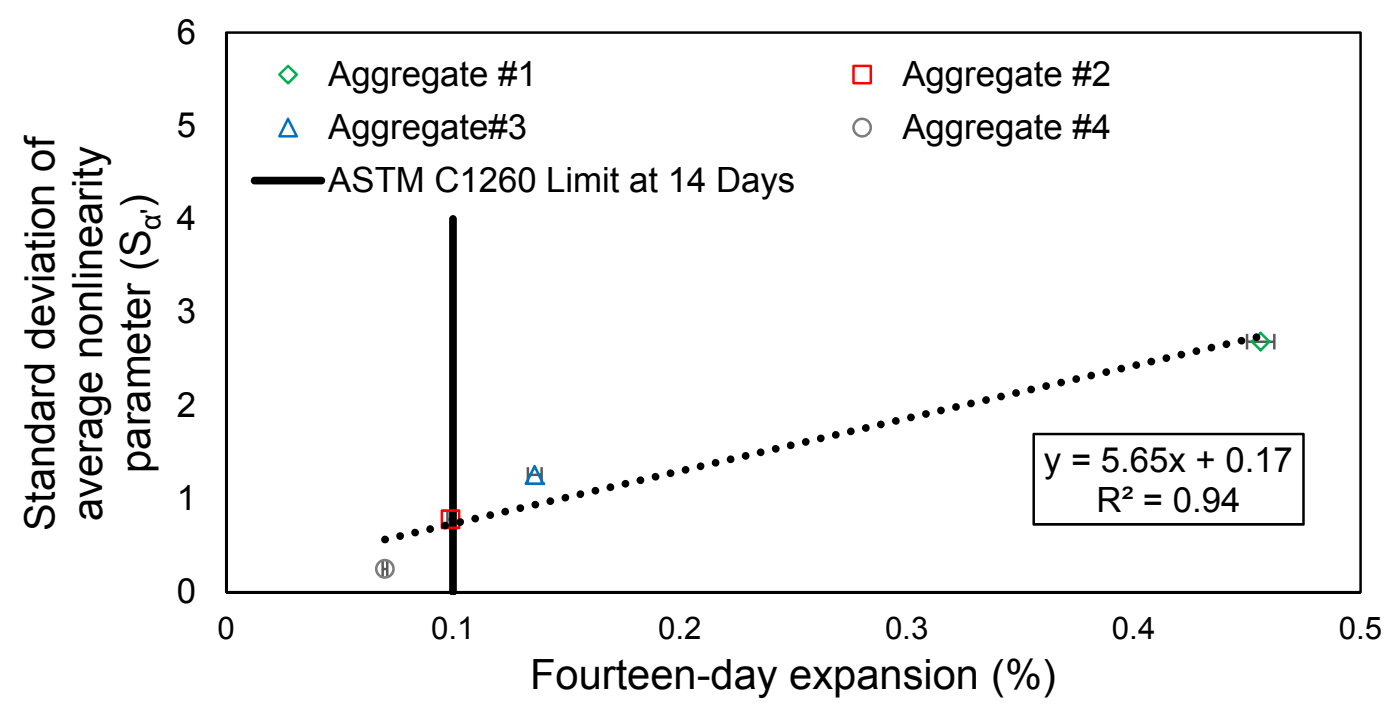

Fig. 11. Correlation between the standard deviation of average nonlinearity parameter and fourteen-day expansion a.) for the sample types of this study, b.) for the data from Chen et al. [8]

\subsubsection{Cumulative average nonlinearity parameter}

The average nonlinearity parameter is used to evaluate the extent of ASR damage in samples. However, damage is due to the progression of ASR during the reaction period, while the duration of measured average nonlinearity parameter is very short compare to that period. In other words, the average nonlinearity parameter is an instantaneous measure of microcracking and damage. Therefore, to evaluate the amount of accumulated damage, the average nonlinearity parameter is integrated [8] over the reaction period. Defining the reaction period is very important; here, the reaction period is defined as the period between day zero of exposure, and the day that a plateau in the trend of the average nonlinearity parameter is reached, where the rate of the reaction slows (for a variety of potential reasons). According to Fig. 10, the end of the reaction period, $R_{p}$, may be selected as day twelve for the Reactive-2 and Non-Reactive samples, and day fourteen for the Reactive-1 samples. Riemann integral evaluates the cumulative average nonlinearity parameter as 


$$
\begin{array}{lc}
C_{\alpha^{\prime}}(i)=0 & i=0 \\
C_{\alpha^{\prime}}(i)=C_{\alpha^{\prime}}(i-1)+\frac{\left[\alpha^{\prime}(i-1)+\alpha^{\prime}(i)\right][t(i)-t(i-1)]}{2} & 1 \leq i \leq R_{p}, \mathrm{R}_{p} \leq 14
\end{array},
$$

where $C_{\alpha^{\prime}}(i)$ is the cumulative average nonlinearity parameter at time $i$, and $t(i)$ time $i$. Furthermore, since the nonlinearity measurements are made daily, $t(i)-t(i-1)$ is equal to one and Eq. (5) is simplified as

$$
\begin{array}{lc}
C_{\alpha^{\prime}}(i)=0 & i=0 \\
C_{\alpha^{\prime}}(i)=C_{\alpha^{\prime}}(i-1)+\frac{\left[\alpha^{\prime}(i-1)+\alpha^{\prime}(i)\right]}{2} & 1 \leq i \leq R_{p}, \mathrm{R}_{p} \leq 14
\end{array}
$$

Furthermore, the standard deviation of cumulative average nonlinearity parameter is achieved according to Eq. (7) as

$$
\begin{array}{lc}
S_{C_{\alpha^{\prime}}}(i)=0 & i=0 \\
S_{C_{\alpha^{\prime}}}(i)=\sqrt{\left[S_{C_{\alpha^{\prime}}}(i-1)\right]^{2}+\left[\frac{S_{\alpha^{\prime}}(i-1)^{2}+S_{\alpha^{\prime}}(i)^{2}}{4}\right]} & 1 \leq i \leq R_{p}, R_{p} \leq 14
\end{array}
$$

Where $S_{C_{\alpha^{\prime}}}(i)$ is the standard deviation of cumulative average nonlinearity parameter at time $i$, and $S_{\alpha^{\prime}}(i)$ the standard deviation of average nonlinearity parameter at time $i$. In addition, Eq. (6) assumes that $C_{\alpha^{\prime}}$ as well as $\alpha^{\prime}$ values are statistically independent of each other.

Fig. 12 shows the cumulative average nonlinearity parameter for each of the three mortar bar types. From day nine until the end of the AMBT, the Reactive-1 samples demonstrate the greatest cumulative average nonlinearity parameter among all sample types. Furthermore, the cumulative average nonlinearity parameter clearly differentiates between the potentially reactive and Non-Reactive samples. By performing hypothesis testing at a p-value of 0.05 , this parameter statistically differentiates between potentially reactive and Non-Reactive samples from day one 
of AMBT, which is the result of distinct averages, and small uncertainties associated with the parameter. Furthermore, the cumulative average nonlinearity parameter of potentially reactive aggregates are similar at some points.

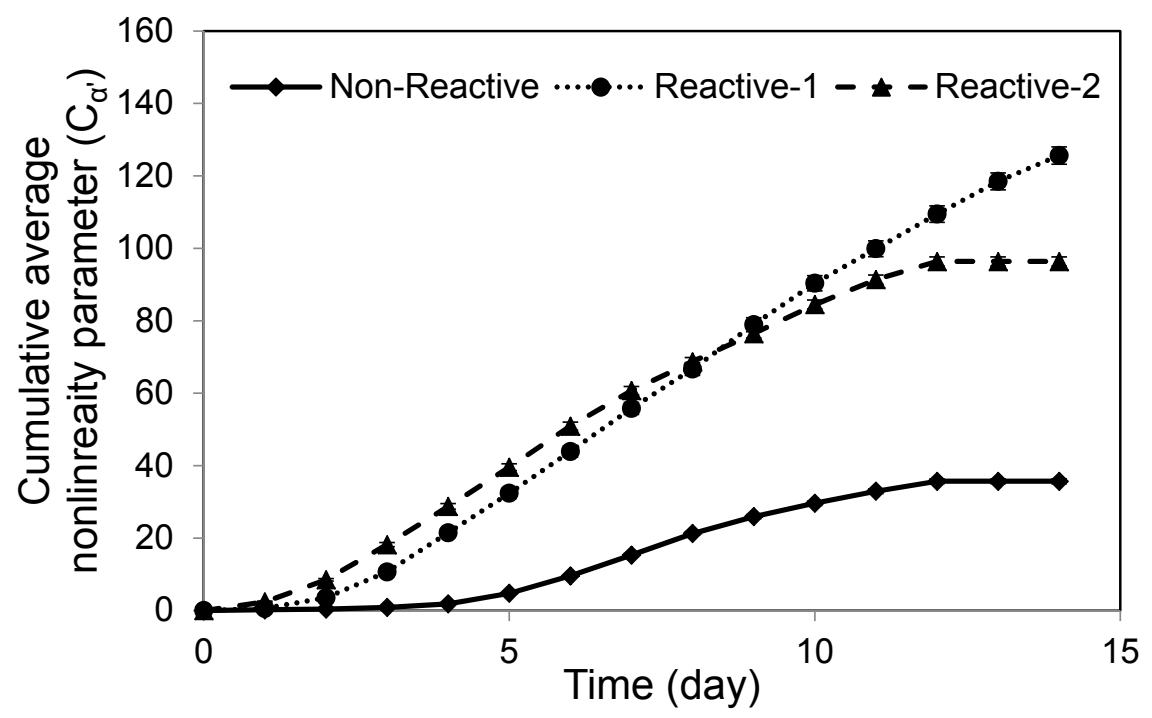

Fig. 12. Temporal cumulative average nonlinearity parameter.

\subsubsection{Hypothesis testing}

To statistically differentiate among the Reactive-1 and Reactive-2 samples hypothesis testing is performed using t-distribution with 58 degrees of freedom and p-value of 0.05 . The number of degrees of freedom is one less than the number of data for each point. To calculate each point of average nonlinearity parameter, at least thirty data points (i.e., impacts) is used. So, the degree of freedom for each point is at least 29. However, since two points are compared, the total degrees of freedom are the sum of their degrees of freedom, which is 58 . The p-value is the probability that potentially reactive samples are not statistically different. In addition, it is assumed that the Reactive-2 samples are statistically independent of the Reactive-1 samples. Thus, those are statistically different at time $i$ if 
$\frac{\left|C_{\alpha_{\text {Reacaive-1 }}^{\prime}}(i)-C_{\alpha_{\alpha_{\text {Reative-2 }}^{\prime}}}(i)\right|}{\sqrt{\left(S_{C_{\alpha_{\text {Reactive-1 }}}}(i)\right)^{2}+\left(S_{C_{\alpha_{\text {Reactive-2 }}}}(i)\right)^{2}}} \geq 2.00$,

Where $C_{\alpha_{\text {Reative-1 }}^{\prime}}(i)$ and $C_{\alpha_{\text {Raative-2 }}^{\prime}}$ (i) are cumulative average nonlinearity parameter for the Reactive-1 and Reactive-2 at time $i$, respectively. Similarly, $S_{C_{a_{\text {Reactive-1 }}}}$ (i) and $S_{C_{a_{\text {Reactive-2 }}}}$ (i) are standard deviation of cumulative average nonlinearity parameter for Reactive- 1 and Reactive- 2 samples at time $i$, respectively. According to the Eq. (8), cumulative average nonlinearity parameter of Reactive-1 and Reactive-2 are statistically different excluding days eight and nine.

\section{Discussion}

All experiments - expansion, NIRAS, microwave measurements, and petrography analysis — successfully differentiate the potentially deleterious aggregate from the innocuous aggregate in mortars subjected to accelerated testing. The experimental results suggest that all four measures can be correlated to some degree; however, the strongest correlations exist between the expansion and nonlinearity, as well as the dielectric properties and the damage evaluated by petrographic analysis. These correlations are considered in further detail.

\subsection{Examining correlations between the cumulative average nonlinearity parameter, expansion and DRI}

The cumulative average nonlinearity parameter shows strong agreement with the AMBT expansion results (Fig. 3) and with the DRI values of potentially reactive samples (Fig. 6). In addition, even the timing of the crossovers of the cumulative average nonlinearity parameter and expansion for the Reactive-1 and Reactive- 2 samples are consistent with one another, occurring between days eight and nine of AMBT exposure. The DRI results, however do not show a 
crossover, but instead show comparable levels of damage for the Reactive-1 and Reactive- 2 samples throughout the duration of testing.

The strong correlation between expansion and the cumulative average nonlinearity parameter is shown in Fig. 13. It is proposed that the correlation originates from the fundamental mechanism by which expansion in a brittle material leads to microcracking and increases material nonlinearity. With ASR, expansion is mainly associated with increased gel volume and pressure. When the tensile capacity is exceeded locally, microcracking results. The total expansion at each age is the cumulative effect of the gel formation and is related then to such microcracking. Furthermore, the extent of microcracking at each time instant is evaluated by the nonlinear acoustic measurements and the cumulative effect of microcracking represented using the cumulative average nonlinearity parameter.

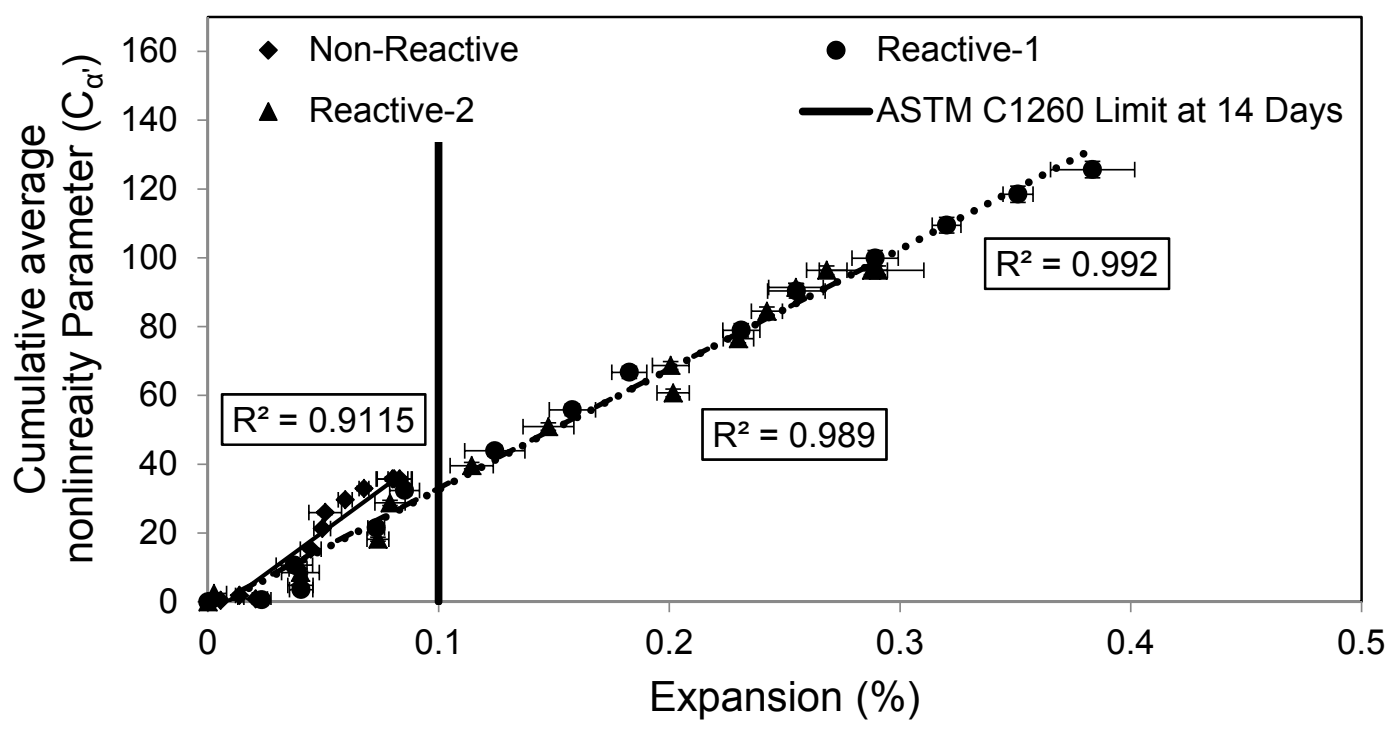

Fig. 13. Correlation between cumulative average nonlinearity parameter and expansion.

The strong coefficient of determination for the Reactive-1 and Reactive- 2 samples supports the linear correlation between the cumulative average nonlinearity parameter and expansion for the reactive aggregates samples studied here. In contrast, the Non-Reactive 
samples have a weaker link between nonlinearity and expansion, and this is confirmed by a weaker coefficient of determination. In addition the Reactive-1 and Reactive- 2 samples have a very similar slope, suggesting a similar causal mechanism contributed to expansion and the cumulative average nonlinearity parameter.

The goodness of linear fit between expansion and the cumulative average nonlinearity parameter of the sample types may represent their potential reactivity. The stronger correlation between expansion and the cumulative average nonlinearity parameter of potential reactive samples originates from comparable rates of increase rate in those measures, which is not the same for the Non-Reactive samples. Fig. 14 shows the relationship between the cumulative average nonlinearity parameter and expansion of Non-Reactive samples. This figure consists of three regions- $\mathrm{A}, \mathrm{B}$, and $\mathrm{C}-$ in which the slope of the linear relationship between the cumulative average nonlinearity parameter and expansion is significantly different. When considering all of the data points in Fig. 14, the significant difference among the slopes between those measures in the $\mathrm{A}, \mathrm{B}$, and $\mathrm{C}$ regions, results in the deviation from the linear relationship between expansion and the cumulative average nonlinearity parameter, and subsequently a lower coefficient of determination. In contrast for the potentially reactive aggregates, the contribution of regions similar to A and B are small and the relationship between those measures is dominated by a region similar to $\mathrm{C}$. Such a behavior results in a stronger correlation between the cumulative average nonlinearity parameter and expansion for the potentially reactive aggregates. 


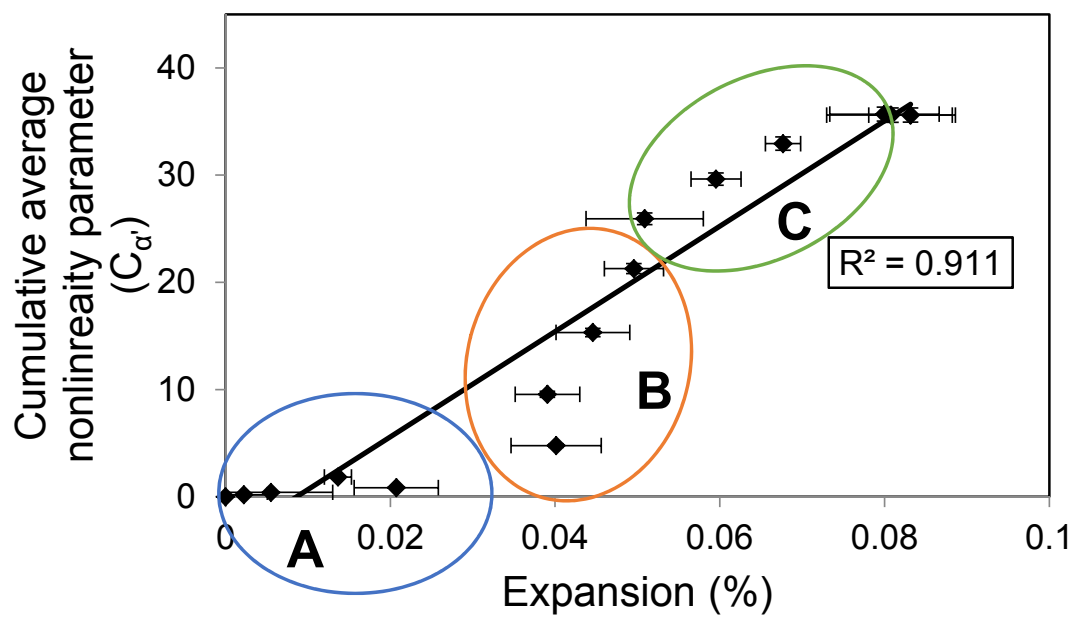

Fig. 14. Correlation between the cumulative average nonlinearity parameter and expansion of Non-Reactive samples.

The cumulative average nonlinearity parameter and the fourteen-day expansion of sample types at the AMBT condition are correlated as shown in Fig. 15. So, the cumulative average nonlinearity parameter not only shows a linear trend with the expansion of each aggregate source (Fig. 13), but it also shows a linear relationship with the expansion of all aggregate sources. These results suggest that a damage criterion for the ASTM C1260 can be proposed. For the aggregates evaluated, a cumulative average nonlinearity of 41 appears to correlate with the AMBT expansion limit of $0.1 \%$, but extensive, further evaluation with a range of aggregate sources, reactive components, and cements is certainly warranted.

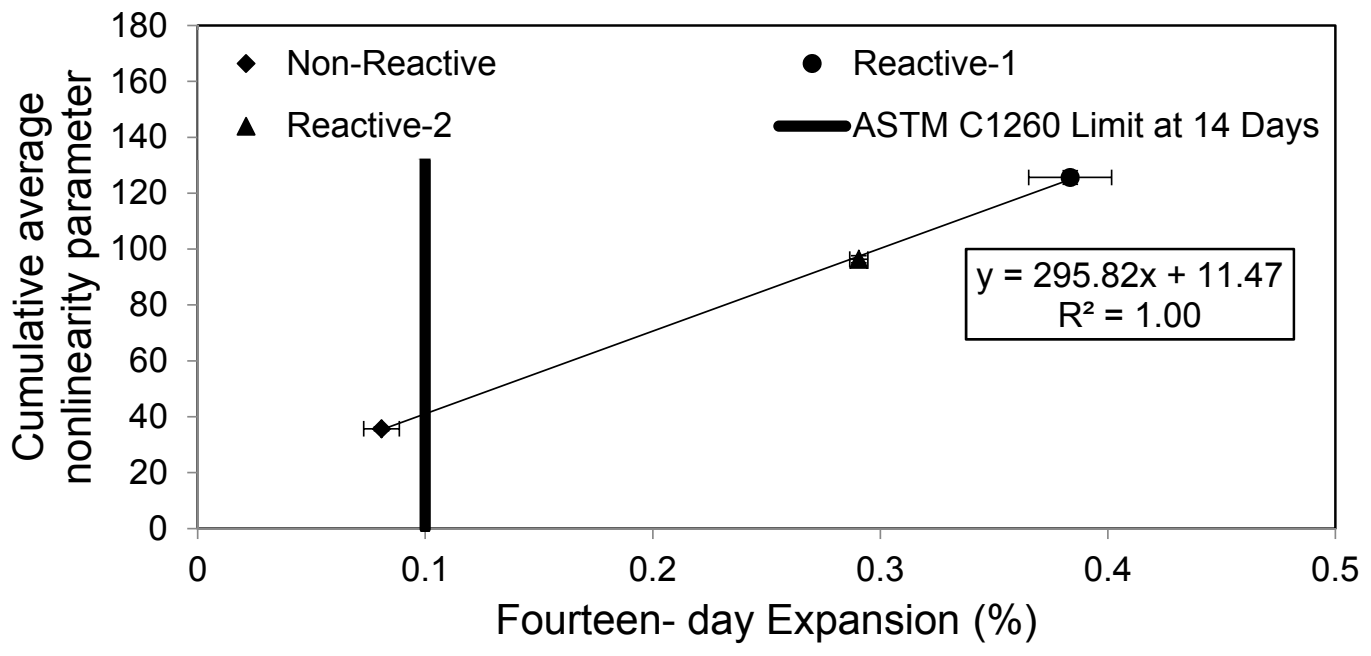


Fig. 15 - Cumulative average nonlinearity parameter and expansion at the day fourteen of AMBT.

While the prior discussion considered a good correlation between expansion and cumulative average nonlinearity parameter (Fig.13), a linear relationship between cumulative average nonlinearity parameter and DRI is also found for reactive aggregates, as shown in Fig. 16. The comparable correlations in Fig.7 and Fig.16 for reactive aggregates originate from the strong correlation between expansion and the cumulative average nonlinearity parameter for those aggregates. In other words, since the cumulative average nonlinearity parameter and expansion are strongly correlated, the correlation of each of them with a third measure (DRI) is similar to that of the other one. In contrast, the correlation in Fig. 7 and Fig. 16 for the NonReactive aggregate are less similar as a result of the weaker correlation between expansion and the cumulative average nonlinearity parameter. Furthermore, this correlation is significantly lower than the correlation for potentially reactive aggregates. While DRI exhibits a linear relationship with cumulative average nonlinearity for the potentially reactive samples, the slope of the best linear fit for the Reactive- 2 samples is greater than that of the Reactive-1 samples. This suggests a larger DRI value should be measured for the Reactive-2 samples than the Reactive-1 samples given the same cumulative average nonlinearity parameter. However, these trends do not agree with the trends in expansion (Fig. 3), where the Reactive-1 samples experience an increase in rate of expansion from day three of AMBT and ultimately have more expansion by the end of the test. 


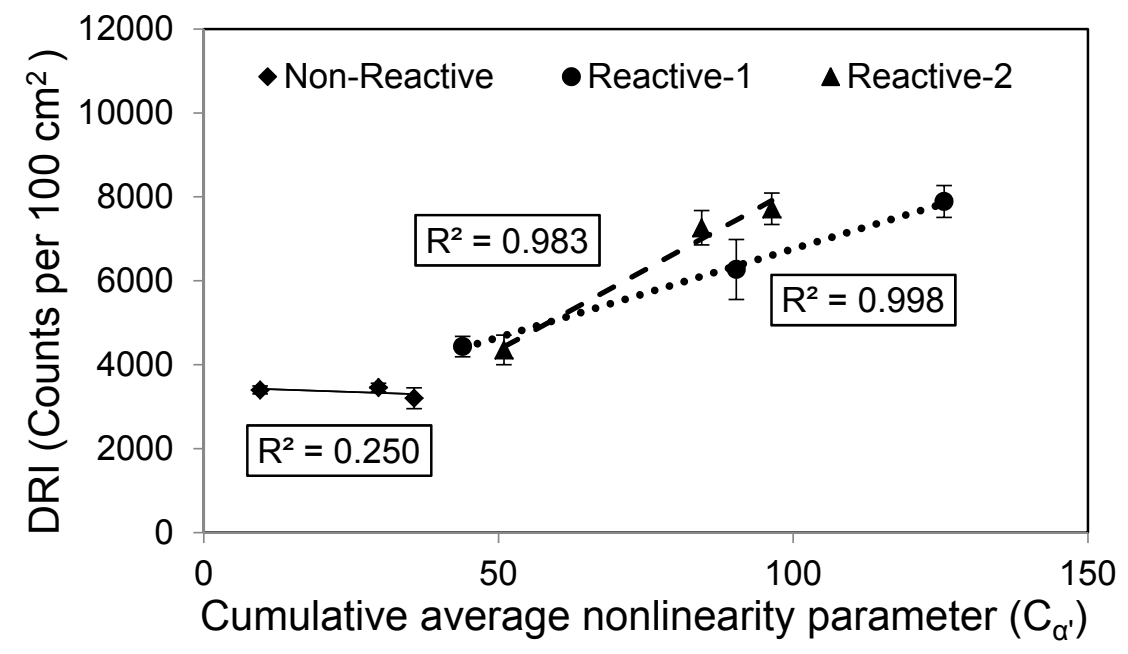

Fig. 16. Correlation between cumulative average nonlinearity parameter and DRI.

\subsection{Examining correlations between relative permittivity, expansion, and DRI}

The correlations between relative permittivity, DRI, and expansion are investigated. Data suggests an agreement between the relative permittivity measured at day 14 of exposure and DRI. Furthermore, while the DRI values do not show a linear relationship with expansion data, the DRI values clearly differentiate between the potentially reactive samples and Non-Reactive ones. These trends are explored in detail below.

\subsubsection{Relative permittivity and DRI}

Fig. 17 shows the relationship between relative permittivity and the DRI values of sample types. By comparing those variables, the potentially reactive samples can be easily distinguished from the Non-Reactive samples. The Reactive-1 and Reactive- 2 samples had both a higher relative permittivity and DRI values than the Non-Reactive samples. This finding suggests an agreement between the relative permittivity and DRI, which may originate from the larger weights that DRI assigns to the defect types associated with ASR gel, and sensitivity of relative permittivity to the 
ASR gel formation. An additional investigation with different aggregate types and at different ages are needed to further develop the relationship between DRI and permittivity more explicitly.

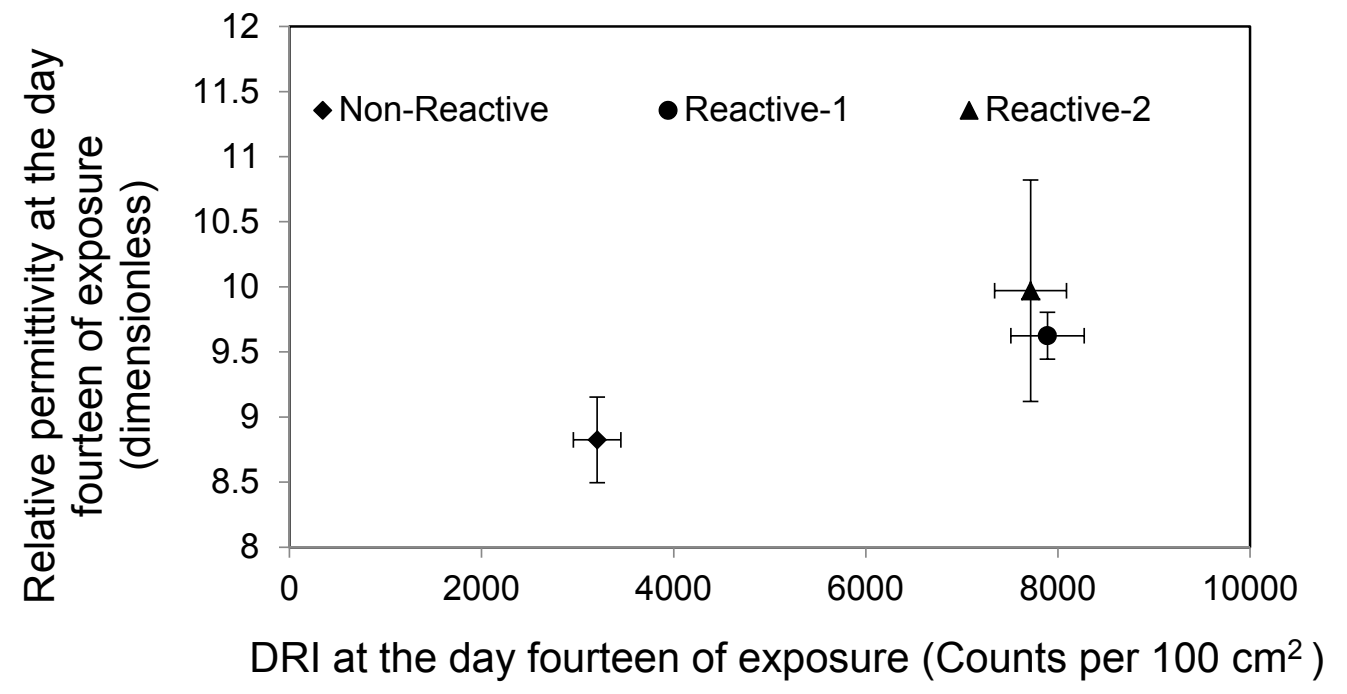

Fig. 17 - Relative permittivity and DRI at the day fourteen of AMBT.

\subsubsection{Relative Permittivity and Expansion}

Fig. 18 shows the relationship between the relative permittivity and the fourteen-day expansion of the sample types. The relative permittivity does not show a linear trend with the fourteen-day expansion. This implies that the amount of gel formed does not necessarily scale with measured expansion. For instance, while the relative permittivity is sensitive to the presence of gel in the air voids and macro-cracks, gel found in these regions may not yield as much expansion as gel confined in smaller voids or microcracks. However, overall, a larger relative permittivity of a sample is associated with greater expansion. Considering the average values, the relative permittivity of the Non-Reactive samples is around 8.8, while it is larger than 9.6 for the potential reactive aggregates. With additional research on a wider range of aggregates, it is proposed that a limit similar to the AMBT expansion limit could be established for the relative permittivity. 


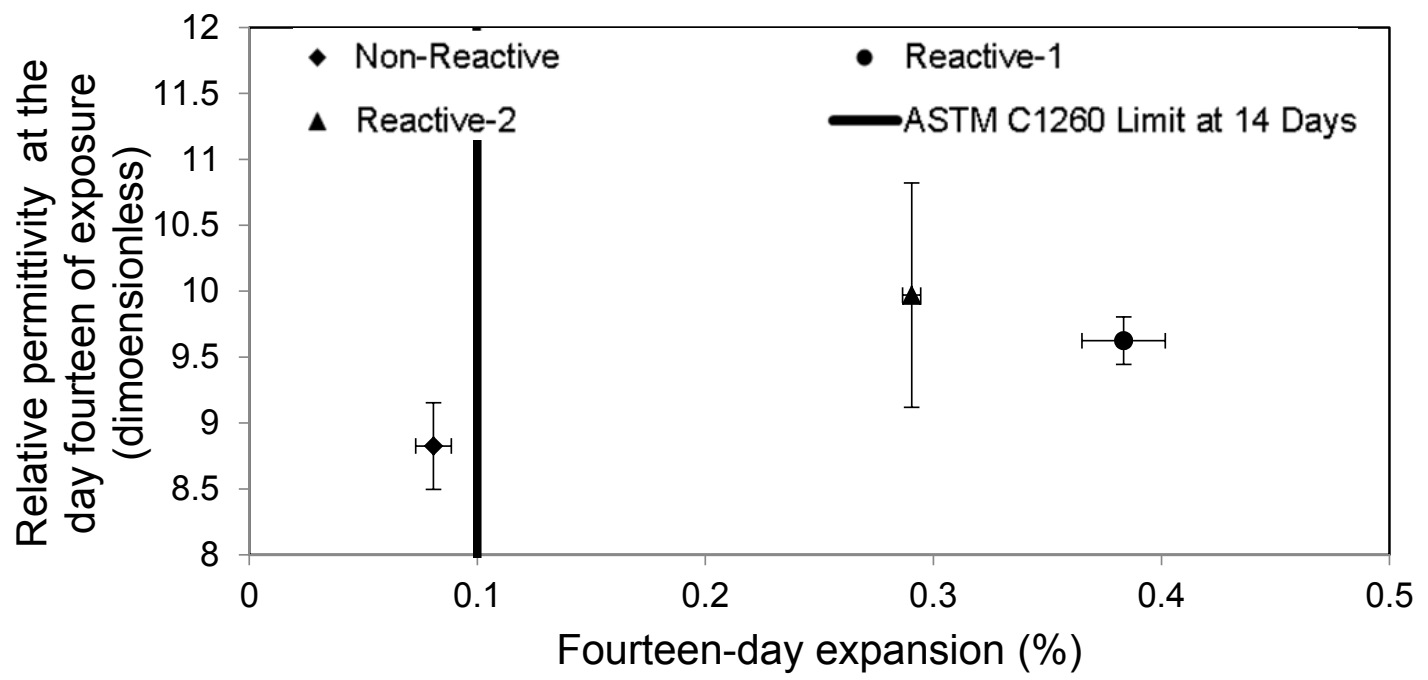

Fig. 18 - Relative permittivity and expansion at the day fourteen of AMBT.

Overall, while this research studies a limited number of aggregates exposed to AMBT, it establishes a new multi-physics-based approach for studying the degradation of cement-based composites, with a specific application to ASR. This research demonstrates that the dielectric properties of ASR-affected cement-based materials are sensitive to the physical and chemical properties of the gel product, which ultimately determines the gel's expansivity and extent and rate of subsequent damage. While expansion and the microstructural indications of damage (via DRI) are measured directly, additional assessment of the effect of microcracking and gel accumulation is provided by nonlinear acoustics. However, it is important to note that the pattern of ASR damage varies with different aggregate sources and environmental conditions. For example, the accelerated test conditions used here may produce gel compositions and behaviors which may or may not be representative of those found in the field, even for these aggregate sources. Therefore, extension of this multi-physics approach for the study of mortars or concretes cast with aggregates varying in mineralogy and reactivity, exposed to both accelerated and field conditions, is needed to further examine the correlations and trends observed in this study. A more exhaustive investigation would provide foundational information about the relationship 
between the gel composition and volume and associated damage, which is valuable for the screening of aggregate sources, for the monitoring of ASR-affected structures, and for the design of appropriate mitigation and repair strategies.

\section{Conclusions}

The results obtained from the four test methods including expansion, nonlinear acoustics, microwave measurements, and quantitative petrographic analysis using the damage rating index (DRI) provide a better picture of how expansion and damage are linked to the gel formation during the AMBT test. The main findings of this study are as follows:

- Among the dielectric properties of the samples, relative permittivity shows higher sensitivity to ASR gel formation, and the samples cast with the potentially reactive aggregates show higher relative permittivity than those prepared with innocuous aggregate.

- DRI values increase over the time of exposure to the AMBT condition, and increase consistently with the expansion data for samples cast with potentially reactive aggregates, while this is not the case for the samples prepared with innocuous aggregate.

- Not only does the cumulative average nonlinearity parameter strongly correlate with the expansion of each sample type, but also this correlation is stronger for samples cast with potentially reactive aggregates than those cast with innocuous aggregate.

- The slope of the linear relationship between the cumulative average nonlinearity parameter and expansion is approximately the same for samples cast with the potentially reactive aggregates, while it is distinct for those prepared with the innocuous aggregate. 
- The correlation between the cumulative average nonlinearity parameter and DRI is stronger for samples prepared with potentially reactive aggregates than those cast with the innocuous aggregate.

- The agreement of the relative permittivity and DRI of sample types may originate from the larger weights that the DRI assigns to the defects containing ASR gel, and the sensitivity of the relative permittivity to the gel presence.

In summary, this study establishes the connection between gel formation, damage, and expansion using these multidisciplinary methods in concert. Combining nonlinear acoustics with microwave measurements provides better insight into the evolution of microcracks and gel formation during the expansion caused by ASR. While the variation of the average nonlinearity parameter originates from the domination of mechanisms that decrease that parameter, such as increased pressure in the microcracks which decreases the interaction of microcracks asperities, to the ones that increase the parameter, such as microcracking, the standard deviation of the average nonlinearity parameter increases consistently with the fourteen-day expansion. This suggests that specimens that experienced larger expansion also experienced greater ASR gel pressure and pressure relief through progressive cracking. In addition, the cumulative average nonlinearity parameter strongly correlates with expansion, indicating it is a good alternative for assessing ASR damage in cement-based materials. On the other hand, microwave testing is useful for assessing the source of microcracking, tracking gel formation, and the accumulation of gel over time. The agreement observed between relative permittivity and DRI, but not with the fourteen-day expansion, demonstrates that the amount of gel formed does not necessarily scale with expansion. Ultimately, while this research has connected gel and damage in a novel and direct manner during an accelerated test, the composition and expansivity of gels found in the 
field may or may not be the same as those formed during the test. Therefore, the application of this multi-physics approach to study mortars and concrete cast with aggregates varying in reactivity and under field exposure conditions can provide further information about the gel properties and volume, and its relationship with microcracking and expansion.

\section{Acknowledgments}

This material is based upon work supported by the National Science Foundation under Grant No. CMMI1234035. Any opinions, findings, and conclusions or recommendations expressed in this material are those of the author(s) and do not necessarily reflect the views of the National Science Foundation.

\section{References}

1. Mehta, P. K., and Monteiro, P. J. M., "Concrete : Microstructure, Properties, and Materials," 3rd. Edition, McGraw-Hill, New York, 2006, 659 pp.

2. Mindess, S., Young, J. F., and Darwin, D., “Concrete," 2nd. Edition, Upper Saddle River, NJ, Prentice Hall, 2003, 644 pp.

3. Chatterji, S., Thaulow, N., and Jensen, A. D., "Studies of Alkali-Silica Reaction. 4. Effect of Different Alkali Salt-Solutions on Expansion," Cement and Concrete Research, V. 17, No. 5, Sep. 1987, pp. 777783.

4. Glasser, L. S. D., and Kataoka, N., "The Chemistry of Alkali-Aggregate Reaction," Cement and Concrete Research, V. 11, No. 1, 1981, pp. 1-9.

5. Marinoni, N., Voltolini, M., Mancini, L., and Cella, F., "Influence of Aggregate Mineralogy on AlkaliSilica Reaction Studied by X-Ray Powder Diffraction and Imaging Techniques," Journal of Materials Science, V. 47, No. 6, Mar. 2012, pp. 2845-2855.

6. Thaulow, N., Jakobsen, U. H., and Clark, B., "Composition of Alkali Silica Gel and Ettringite in Concrete Railroad Ties: SEM-EDX and X-Ray Diffraction Analyses," Cement and Concrete Research, V. 26, No. 2, Feb. 1996, pp. 309-318.

7. Rashidi, M.,Paul, A., Kurtis, K.E., "Investigating the Role of Alkalis in the Alkali-Silica Reaction (ASR) Gel," Cement and Concrete Research, (In preparation).

8. Chen, J., Jayapalan, A. R., Kim, J.-Y., Kurtis, K. E., and Jacobs, L. J., "Rapid Evaluation of AlkaliSilica Reactivity of Aggregate Using a Nonlinear Resonance Spectroscopy Technique," Cement and Concrete Research, V. 40, No. 6, Jun. 2010, pp. 914-923. 
9. Sargolzahi, M., Kodjo, S.A., Rivard, P. and Rhazi, J. "Effectiveness of nondestructive testing for the evaluation of alkali-silica reaction in concrete," Construction and Building Materials, V. 24, No.8, 2010, pp.1398-1403.

10. Saint-Pierre, F., Rivard, P., and Ballivy, G., "Measurement of Alkali-Silica Reaction Progression by Ultrasonic Waves Attenuation," Cement and Concrete Research, V. 37, No. 6, Jun. 2007, pp. 948-956.

11. Lesnicki, K. J., Kim, J. Y., Kurtis, K. E., and Jacobs, L. J., "Characterization ASR Damage in Concrete Using Nonlinear Impact Resonance Acoustic Spectroscopy Technique," NDT \& E International, V. 44, No. 8, Dec. 2011, pp. 721-727.

12. "Standard Specifications for Transportation Materials and Methods of Sampling and Testing, 34th Edition and AASHTO Provisional Standards, ”Edition, 2014.

13. "Standard Test Method for Determination of Length Change of Concrete Due to Alkali-Silica Reaction. ," American Society for Testing Materials, V. 4, No.2, Annual Book of ASTM Standards, 2008, pp. 682-688.

14. Nazarov, V.E., Ostrovsky, L.A., Soustova, I.A. and Sutin, A.M., "Nonlinear acoustics of microinhomogeneous media," Physics of the Earth and Planetary Interiors, V. 50, No. 1, 1988, pp.65-73.

15.Van Den Abeele, K.E., Johnson, P.A., Guyer, R.A. and McCall, K.R. "On the quasi-analytic treatment of hysteretic nonlinear response in elastic wave propagation," The Journal of the Acoustical Society of America, V. 101, No. 4, 1997, pp.1885-1898.

16. Van Den Abeele, K.A., Johnson, P.A. and Sutin, A., "Nonlinear elastic wave spectroscopy (NEWS) techniques to discern material damage, part I: nonlinear wave modulation spectroscopy (NWMS)," Research in nondestructive evaluation, V. 12, No.1, 2000, pp.17-30.

17. Van Den Abeele, K.A., Carmeliet, J., Ten Cate, J.A. and Johnson, P.A., "Nonlinear elastic wave spectroscopy (NEWS) techniques to discern material damage, Part II: Single-mode nonlinear resonance acoustic spectroscopy," Journal of Research in Nondestructive Evaluation, V. 12 No. 1, 2000, pp.31-42.

18. Peer, S., Kurtis, K. E., and Zoughi, R., "An Electromagnetic Model for Evaluating Temporal Water Content Distribution and Movement in Cyclically Soaked Mortar," IEEE Transactions on Instrumentation and Measurement, V. 53, No. 2, Apr. 2004, pp. 406-415.

19. Bois, K. J., Benally, A. D., Nowak, P. S., and Zoughi, R., "Cure-State Monitoring and Water-toCement Ratio Determination of Fresh Portland Cement-Based Materials using Near-Field Microwave Techniques," IEEE Transactions on Instrumentation and Measurement, V. 47, No. 3, Jun. 1998, pp. 628637.

20. Peer, S., Case, J. T., Gallaher, E., Kurtis, K. E., and Zoughi, R., "Microwave reflection and dielectric properties of mortar subjected to compression force and cyclically exposed to water and sodium chloride solution," Instrumentation and Measurement, IEEE Transactions on, V. 52, No. 1, 2003, pp. 111-118.

21. Donnell, K. M., Hatfield, S., Zoughi, R., and Kurtis, K. E., "Wideband Microwave Characterization of Alkali-Silica Reaction (ASR) Gel in Cement-Based Materials," Materials Letters, V. 90, Jan 1. 2013, pp. 159-161.

22. Donnell, K. M., Zoughi, R., and Kurtis, K. E., "Demonstration of Microwave Method for Detection of Alkali-Silica reaction (ASR) Gel in Cement-Based Materials," Cement and Concrete Research, V. 44, No. 0, February. 2013, pp. 1-7. 
23. Hashemi, A., Donnell, K. M., Zoughi R., and Kurtis., K. E. "Effect of humidity on dielectric properties of mortars with alkali-silica reaction (ASR) gel." Instrumentation and Measurement Technology Conference (I2MTC), 2015 IEEE International, pp. 1502-1506.

24. Ulaby, F.T., Moore, R.K., Fung, A.K., "Microwave remote sensing: Active and passive, vol. III, volume scattering and emission theory, advanced systems and applications." Inc., Dedham, Massachusetts 1986.

25. McNeal, M. P., Jang, S. J., and Newnham, R. E., "The Effect of Grain and Particle Size on the Microwave Properties of Barium Titanate (Batio3)," J Appl Phys, V. 83, No. 6, Mar. 1998, pp. 32883297.

26. "Standard Test Method for Potential Alkali Reactivity of Aggregate (Mortar-Bar Method)." West Conshohocken, PA: ASTM International, 2007.

27. Boukari, Y., Bulteel, D., Rivard, P. and Abriak, N.E., 2015. "Combining nonlinear acoustics and physico-chemical analysis of aggregates to improve alkali-silica reaction monitoring," Cement and Concrete Research, 67, pp.44-51.

28. Stauffer, J. D., Woodward, C. B., and White, K. R., "Nonlinear Ultrasonic Testing with Resonant and Pulse Velocity Parameters for Early Damage in Concrete," ACI Mater J, V. 102, No. 2, Mar-Apr. 2005, pp. 118-121.

29. Kesner, K., Sansalone, M., and Poston, R. W., "Detection of Distributed Damage in Concrete Using Transient Stress Waves,” Edition, V. 3400, Spie-Int Soc Optical Engineering, Bellingham, 1998.

30. Guyer, R. A., and Johnson, P. A., "Nonlinear Mesoscopic Elasticity: Evidence for a New Class of Materials," Physics Today, V. 52, No. 4, Apr. 1999, pp. 30-36.

31. Chen, J., Lesnicki, K. J., Kurtis, K. E., Kim, J. Y., and Jacobs, L. J., "NDT Techniques for Characterizing Alkali-Silica Reaction in Standard Concrete Specimens - A Review," in Review of Progress in Quantitative Nondestructive Evaluation, Vols 30a and 30b. V.1335, 2011, pp. 1275-1282.

32. Leśnicki, K. J., Kim, J. Y., Kurtis, K. E., and Jacobs, L. J., “Assessment of alkali-silica reaction damage through quantification of concrete nonlinearity," Materials and structures, V. 46, No. 3, Mar. 2013, pp.497-509.

33. Rashidi, M., Kim, J. Y., Jacobs, L. J., and Kurtis, K. E., "Insights into alkali-silica reaction damage in mortar through acoustic nonlinearity, " 42nd ANNUAL REVIEW OF PROGRESS IN QUANTITATIVE NONDESTRUCTIVE EVALUATION .

34. Rashidi, M. M. N., Paul, A., Kim, J. Y., Jacobs, L. J., \& Kurtis, K. E. "Evaluation of nonlinear impact resonance spectroscopy method for detecting delayed ettringite formation," $41^{\text {ST }}$ ANNUAL REVIEW OF PROGRESS IN QUANTITATIVE NONDESTRUCTIVE EVALUATION, V. 34, Vol. 1650, pp. 1632-1640 Mar. 2015.

35. Rashidi, M., Paul, A., Kim, J. Y., Jacobs, L. J., \& Kurtis, K. E. "Characterization of delayed ettringite formation using nonlinear impact resonance spectroscopy method," Cement and Concrete composites (Submitted).

36. Rivard, P., Fournier, B., and Ballivy, G., "The Damage Rating Index Method for ASR Affected Concrete - A Critical Review of Petrographic Features of Deterioration and Evaluation Criteria," Cement Concrete and Aggregate, V. 24, No. 2, Dec. 2002, pp. 81-91. 
37. Grattan-Bellew, P. E., "Laboratory Evaluation of Alkali-Silica Reaction in Concrete from Saunders Generating-Station," ACI Mater J, V. 92, No. 2, Mar-Apr. 1995, pp. 126-134.

38. Rivard, P., and Ballivy, G., "Assessment of the expansion related to alkali-silica reaction by the Damage Rating Index method." Construction and Building Materials, V. 19, No. 2, Mar. 2005, pp. 8390.

39. Natesaiyer, K. C., and Hover, K. C., "Further Study of an In Situ Identification Method for AlkaliSilica Reaction-Products in Concrete," Cement and Concrete Research, V. 19, No. 5, Sep. 1989, pp. 770778.

40. "Standard Practice for Petrographic Examination of Hardened Concrete," West Conshohocken, PA: ASTM International, 2013.

41. Bois, K. J., Handjojo, L. F., Benally, A. D., Mubarak, K., \& Zoughi, R. "Dielectric plug-loaded twoport transmission line measurement technique for dielectric property characterization of granular and liquid materials," IEEE transactions on instrumentation and measurement, V. 48, No. 6, Dec. 1999, pp. 1141-1148.

42. Jackson, S., "Research methods and statistics: A critical thinking approach," ed: Cengage Learning, 2011.

43. Hashemi A., Rashidi M., Kurtis K.E., Donnell K.M., and Zoughi R. "Microwave Dielectric Properties Measurements of water glasses," Materials letters 2015.

44. Kirkpatrick, R. J., Kalinichev, A. G., Hou, X., and Struble, L. "Experimental and molecular dynamics modeling studies of interlayer swelling: water incorporation in kanemite and ASR gel," Materials and Structures, V. 38, No. 4, May 2005, pp. 449-458.

45. Hashemi, A., Horst, M., Kurtis, K. E., Donnell, K. M., and Zoughi, R. "Comparison of Alkali-Silica Reaction Gel Behavior in Mortar at Microwave Frequencies," IEEE transactions on instrumentation and measurement, 2014.

46. V. Aleshin, K. Van Den Abeele, "Microcontact-based theory for acoustics in microdamaged materials, Journal of the Mechanics and Physics of Solids," V.55, 2007, pp. 366-390. 